\title{
Analytic and numerical models of the 3D multipolar magnetospheres of pre-main sequence stars
}

\author{
Scott G. Gregory ${ }^{1, \star}$ and Jean-François Donati ${ }^{2}$ \\ 1 California Institute of Technology, MC 249-17, Pasadena, 91101 CA, U. S. A. \\ 2 IRAP-UMR 5277, CNRS \& Univ. de Toulouse, 14 Av. E. Belin, F-31400 Toulouse, France
}

Received 20 September 2011, accepted 19 October 2011

Published online later

Key words stars: magnetic fields - stars: general - stars: pre-main sequence

Traditionally models of accretion of gas on to T Tauri stars have assumed a dipole stellar magnetosphere, partly for simplicity, but also due to the lack of information about their true magnetic field topologies. Before and since the first magnetic maps of an accreting T Tauri star were published in 2007 a new generation of magnetospheric accretion models have been developed that incorporate multipole magnetic fields. Three-dimensional models of the large-scale stellar magnetosphere with an observed degree of complexity have been produced via numerical field extrapolation from observationally derived T Tauri magnetic maps. Likewise, analytic and magnetohydrodynamic models with multipolar stellar magnetic fields have been produced. In this conference review article we compare and contrast the numerical field extrapolation and analytic approaches, and argue that the large-scale magnetospheres of some (but not all) accreting T Tauri stars can be well described by tilted dipole plus tilted octupole field components. We further argue that the longitudinal field curve, whether derived from accretion related emission lines, or from photospheric absorption lines, provides poor constrains on the large-scale magnetic field topology and that detailed modeling of the rotationally modulated Stokes V signal is required to recover the true field complexity. We conclude by examining the advantages, disadvantages and limitations of both the field extrapolation and analytic approaches, and also those of magnetohydrodynamic models.

\section{Introduction}

The original models of magnetospheric accretion on to a $\mathrm{T}$ Tauri star assumed the stellar magnetosphere was a dipole (Königl 1991; Collier Cameron \& Campbell 1993; Hartmann et al 1994; Shu et al 1994). This assumption was made partly for simplicity and partly due to a lack of available information about the true large-scale magnetic field topology of such stars. None-the-less dipole magnetospheric models have proved successful in reproducing many observational signatures, for example, the profiles and absorption components of accretion related emission lines (e.g. Hartmann et al 1994; Kurosawa et al 2006). Since the middle of the 1990s several two dimensional magnetohydrodynamic (MHD) models have been presented (e.g. Goodson et al 1997; Miller \& Stone 1997; Romanova et al 2002; Küker et al 2003; von Rekowski \& Piskunov 2006; Bessolaz et al 2008; Zanni \& Ferreira 2009), as well as some three dimensional (3D) simulations with tilted dipole magnetospheres (Romanova et al 2003, 2004; Orlando et al 2011) - see Gregory et al (2010) for a review.

Dipolar accretion models are not without their critiques (e.g. Safier 1998), and dropping the dipole assumption can significantly affect the structure of accretion columns within the magnetosphere (Gregory et al 2006a), the locations of hot spots at the base of accretion funnels (Gregory et al

\footnotetext{
^ Corresponding author: e-mail: sgregory@caltech.edu
}

2005; Mohanty \& Shu 2008), and the density of the gas in the flow (Gregory et al 2007). High order field components may even play a dominate role in the physics of the gas inflow as the accretion columns approach the star (Adams \& Gregory 2011).

Through the analysis of Zeeman broadened lines in intensity spectra T Tauri stars have been found to host surface averaged magnetic fields of order a kilo-Gauss (see JohnsKrull 2007 and references there-in). Provided that the surface fields are associated with a stellar magnetosphere that is sufficiently globally ordered then such strong fields are able to disrupt the circumstellar disk at a distance of a few stellar radii, as required by the magnetospheric accretion scenario (Königl 1991). There appears to be little variation in the average surface field between sources, and between accreting and non-accreting stars, although intriguingly the average (unsigned) magnetic flux appears to be less, on average, for stars in older star forming regions (Yang \& Johns-Krull 2011).

Spectropolarimetry provides a method of probing the magnetic field topology. By measuring the circular polarization signal in magnetically sensitive photospheric absorption lines, and in accretion related emission lines, information can be obtained about the surface fields of T Tauri stars. Zero net polarization signals have often been measured in photospheric absorption lines (e.g. Valenti \& Johns-Krull 2004). As such lines form essentially uniformly across the entire visible stellar surface, this suggests that $\mathrm{T}$ Tauri stars 
host complex surface fields with roughly equal amounts of positive and negative field regions. However, often accretion related emission lines, such as HeI $5876 \AA$, show strong levels of circular polarization (e.g. Johns-Krull et al 1999), with the signal modulated on a timescale equal to the photometrically determined rotation period of the star (Valenti \& Johns-Krull 2004). This suggests that for many T Tauri stars magnetospheric accretion occurs predominantly into strong single polarity spots on the surface the star. However, such studies which are based on measuring the phase modulated stellar disk averaged longitudinal field component, cannot constrain the field topology of the star beyond the interpretation that $\mathrm{T}$ Tauri stars have complex surface fields, and somewhat simpler large scale fields.

In 2007 the first magnetic maps of the large-scale field of an accreting $\mathrm{T}$ Tauri star were published (Donati et al 2007). At the time of writing magnetic maps have now been produced for 10 accreting T Tauri stars namely V2129 Oph, BP Tau, V2247 Oph, AA Tau, TW Hya, both stars of the close binary V4046 Sgr, CR Cha, CV Cha and MT Ori (Donati et al 2007, 2008b, 2010a,b, 2011a,b,c; Hussain et al 2009; Skelly et al 2011). They are produced by monitoring the rotational modulation of the circular polarization signal over a complete stellar rotation cycle, and in practise several, detected in both photospheric absorption and accretion related emission lines. As the polarization signal in individual photospheric absorption lines is vanishingly small cross-correlation techniques (such as Least-Squares Deconvolution (LSD); Donati et al 1997) are employed in order to extract information from as many of the spectral lines as possible. The complete process, known as Zeeman-Doppler imaging, see Donati (2001) for a review and Donati et al (2010b) for its specific application to accreting T Tauri stars, simultaneously reconstructs maps of the radial, azimuthal and meridional field components at the stellar surface, as well as a brightness map (the surface distribution of cool spots) and a map of excess emission in accretion related lines (the surface distribution of accretion hot spots). Due to the flux cancellation effect that affects all polarization measurements, the magnetic maps only contain information about the large scale field topology. Once the magnetic maps have been derived the field components can be written in terms of spherical harmonics (e.g. Donati et al 2006b). From the maps the coefficients of the spherical harmonic decomposition can be calculated, and from these the polar strength of the each of the multipole moments along with their tilts relative to the stellar rotation axis, and the phase which they are tilted towards, can be determined. A description of the observational process involved in constructing stellar magnetic maps via Zeeman-Doppler imaging, as well as the assumptions and limitations, can be found elsewhere in this volume in the paper of G. A. J. Hussain, to which readers are referred for further details (see also Donati \& Landstreet 2009).

Most of the published magnetic maps have been obtained as part of the Magnetic Protostars \& Planets (MaPP) program. This ongoing large program consists of 690 hours of observing with the ESPaDOnS (Echelle SpectroPolarimetric Device for the Observation of Stars; Donati et al 2006a) instrument at the Canada-France-Hawai'i Telescope (CFHT) between 2008 and 2012, 1130 hours with the NARVAL spectropolarimeter (Aurière 2003) at the Télescope Bernard Lyot (TBL) in the Pyrenées, and additional ground and space based observations (e.g. XMM-Newton, Chandra and HST) for select sources.

In this paper we discuss the results from the ongoing MaPP program and present magnetic maps for some accreting $\mathrm{T}$ Tauri stars in section 2 We demonstrate that the large scale field of some (but not all) $\mathrm{T}$ Tauri stars are well described by fields consisting of a tilted dipole plus a tilted octupole component. We review the current 3D field extrapolation and analytic models of their magnetospheres in section 3 , and argue that use of the stellar disk averaged longitudinal field component alone provides poor constrains on the magnetic field topology. We conclude in section 4 by comparing both approaches to one another, and also to magnetohydrodynamic models, discussing the advantages, disadvantages and limitations of each.

\section{Surface magnetic maps}

$\mathrm{T}$ Tauri stars are found to host multipolar magnetic fields, but the field topology varies between stars and over time for the same star. All appear to have dipole components that are strong enough to disrupt their circumstellar disks. Although their large scale fields are non-dipolar, they are often simpler than the highly complex fields of rapidly rotating zero-age main sequence stars (e.g. Donati et al 2003). This result is not a phase coverage effect, as demonstrated by Hussain et al (2009). Some T Tauri stars stars host simple axisymmetric fields with strong kilo-Gauss dipole components (e.g AA Tau and BP Tau). Others host dominantly axisymmetric fields but field modes of higher order than the dipole are found to be the most dominant (typically, but not exclusively, the octupole component), and their dipole components may be strong or weak (e.g. TW Hya, V2129 Oph and MT Ori). Others host complex magnetic fields that are highly non-axisymmetric with very weak dipole components (< $0.1 \mathrm{kG}$; e.g. V4046 Sgr AB, CR Cha and CV Cha). There is strong link with stellar internal structure where fully convective stars close to the end of the fully convective phase host simple fields with strong dipole components. The dipole component decays rapidly with the development of a radiative core, although the large scale field appears to retain its axisymmetry until the core occupies a substantial volume of the star. Empirically once the mass of the core exceeds about $40 \%$ of the stellar mass, the field becomes highly complex and the dipole component is weak (see Gregory et al 2012 for full details of how $\mathrm{T}$ Tauri magnetic topologies vary with stellar internal structure). There is also tentative evidence for a bi-stable dynamo process operating amongst the lowest mass fully convective 


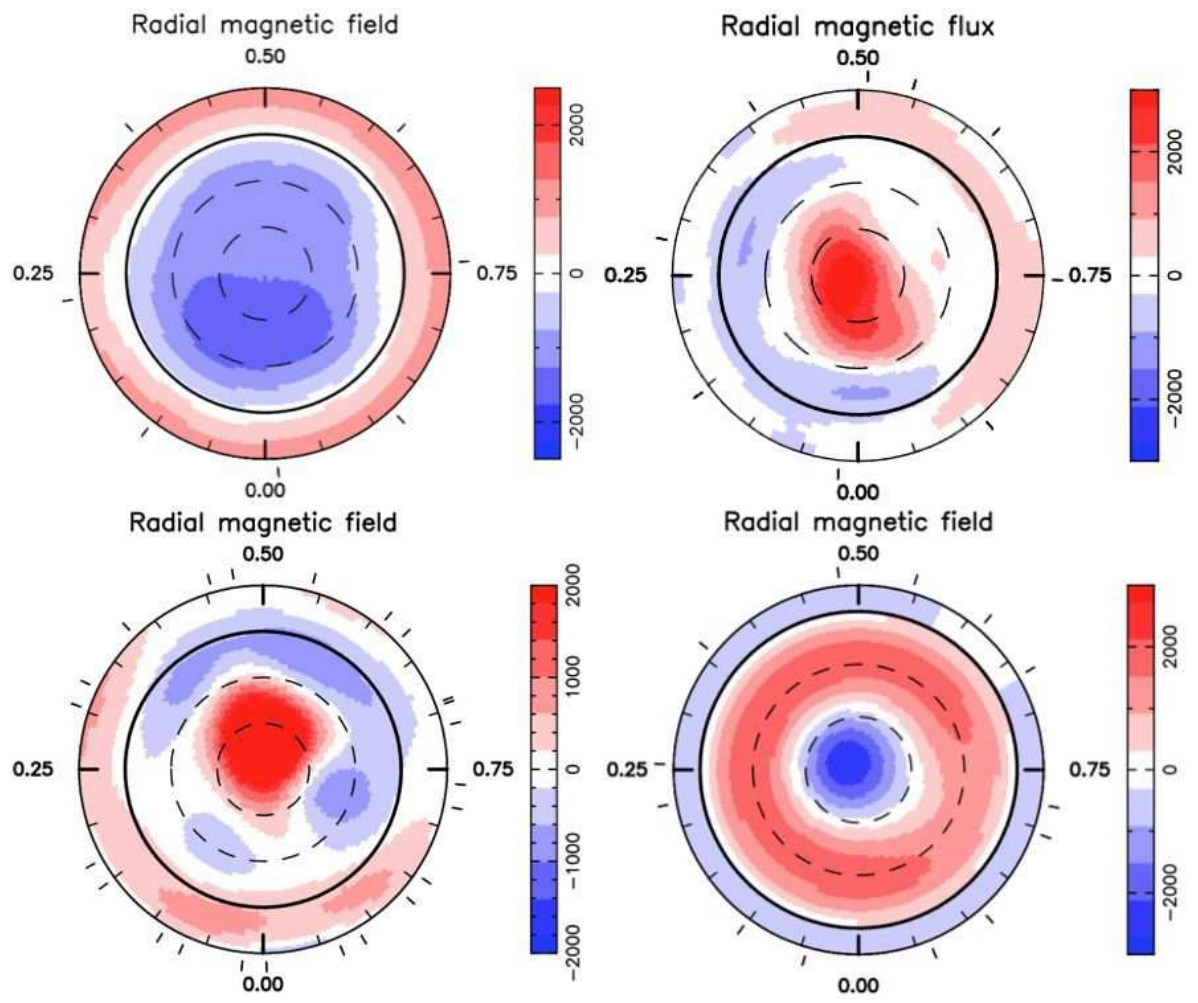

Fig. 1 Magnetic maps showing only the radial component of the field of four accreting $T$ Tauri stars derived from Zeeman-Doppler imaging. The large-scale fields of AA Tau in January 2009 (upper left; Donati et al 2010b), BP Tau in February 2006 (upper right; Donati et al 2008), V2129 Oph in July 2009 (lower left; Donati et al 2011a) and TW Hya in March 2010 (lower right; Donati et al 2011b) are multipolar, but well described as dipole-octupole composite fields with different polar strengths and moment tilts for each star (see Table1). The maps are shown in flattened polar projection with positive/negative field regions in red/blue and labeled in Gauss. The stellar rotation pole is in the middle of each map, the dotted lines are lines of constant latitude separated by $30^{\circ}$ with the equator as the bold circle. The numbers and tick marks around the circumference denote phase and phases of observation respectively. Other T Tauri stars possess more complex large-scale magnetic fields, see Figure 2

Table 1 Polar field strengths of the dipole and octupole components, their tilts relative to the visible stellar rotation pole $(\beta)$, and the rotation phase that they are tilted towards $(\Phi)$ for the stars show in Fig. 10 The values listed for BP Tau were derived using an experimental version of the magnetic imaging code and have recently been revised from the values shown here (see footnote 1 for details). The fields of TW Hya and V2129 Oph were significantly different at other observing epochs.

\begin{tabular}{ccccccccc}
\hline Star & Date & $\begin{array}{c}B_{\text {dip }} \\
(\mathrm{kG})\end{array}$ & $\beta_{\text {dip }}$ & $\Phi_{\text {dip }}$ & $\begin{array}{c}B_{\text {oct }} \\
(\mathrm{kG})\end{array}$ & $\beta_{\text {oct }}$ & $\Phi_{\text {oct }}$ & $B_{\text {oct }} / B_{\text {dip }}$ \\
\hline AA Tau & Jan09 & 1.7 & $170^{\circ}$ & 0.65 & 0.5 & $10^{\circ}$ & 0.50 & 0.29 \\
BP Tau & Feb06 & 1.2 & $20^{\circ}$ & 0.65 & 1.6 & $10^{\circ}$ & 0.15 & 1.33 \\
V2129 Oph & Ju109 & 1.0 & $15^{\circ}$ & 0.40 & 2.2 & $20^{\circ}$ & 0.50 & 2.20 \\
TW Hya & Mar10 & 0.7 & $5^{\circ}$ & 0.25 & 2.8 & $>175^{\circ}$ & 0.85 & 4.00 \\
\hline
\end{tabular}

T Tauri stars, where we expect some to host complex fields, and others simple fields with strong dipole components like the more massive fully convective pre-main sequence stars. This behavior mirrors the magnetic topology trends already found for main sequence M-dwarfs on either side of the fully convective limit (Donati et al 2008a; Morin et al 2008, 2010, 2011).

Figure 1 shows four examples of magnetic maps of accreting $\mathrm{T}$ Tauri stars. Only the radial field component is shown, and we have deliberately selected those stars which have large-scale field topologies that are somewhat simple and well described as dipole-octupole fields, as these will be useful for our analytic work in section 3.2. Other stars have significantly more complex fields, see Figure 2. Maps of the other field components along with brightness and excess accretion related emission maps can be found in the appropriate papers. 

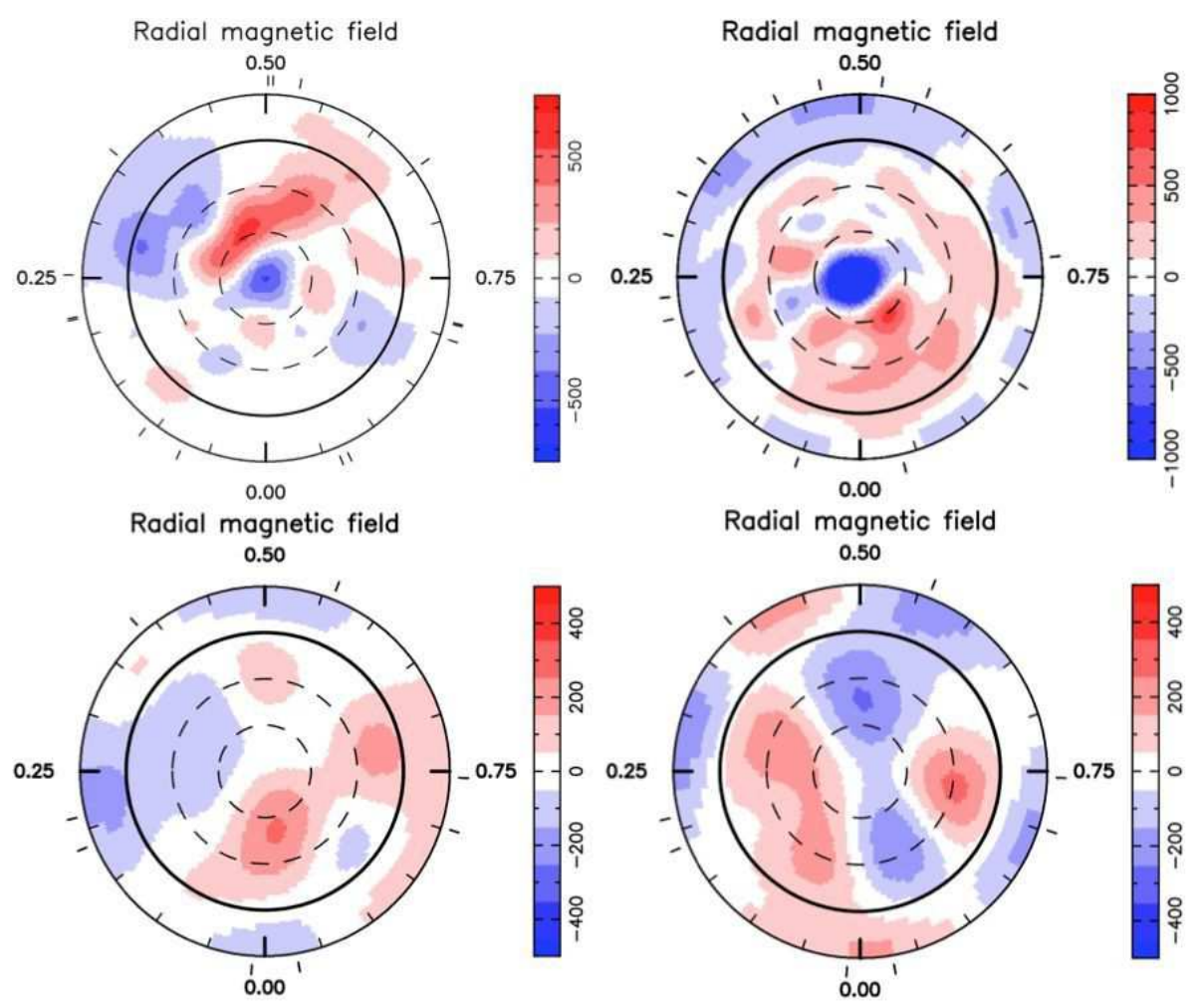

Fig. 2 As Figure 1 but showing magnetic maps of accreting T Tauri stars with large scale fields that are not well described as dipole-octupole composite fields; V2247 Oph in July 2008 (upper right; Donati et al 2010a), MT Ori in December 2008 (upper left; Skelly et al 2011), and both stars of the close binary V4046 Sgr in September 2009 (Donati et al 2011c), the primary (lower left) and the secondary (lower right). The field of MT Ori is similar to that of TW Hya and V2129 Oph in that its field is dominantly axisymmetric but field modes of higher order than the dipole dominate (in this case there are roughly equal contributions from the octupole $[\ell=3]$, the dotriacontapole $[\ell=5]$ and the $\ell=7$ field modes). The fields of the other stars are complex and non-axisymmetric with weak dipole components, as are the fields of CR Cha and CV Cha (Hussain et al 2009; maps not shown here).

The four stars shown in Figure 1 have dipole and octupole components of differing polar strength, and tilts relative to the stellar rotation axis, as summarized in Table 11$]$ In this work we define the tilt $\beta$ of the dipole or octupole moment to be that of the main positive pole relative to the visible rotation pole of the star. Therefore a star which has the main negative pole of the dipole coincident with the visible rotation pole would have $\beta_{d i p}=180^{\circ}$, and likewise $\beta_{\text {oct }}=180^{\circ}$ would correspond to the main negative pole of the octupole coinciding with the visible rotation pole. This is applicable to AA Tau and TW Hya which are close to a configuration with anti-parallel dipole and octupole component at the epochs listed in Table 1 An alternative notation would be to define the tilts of the components according to whichever main magnetic pole was in the visible hemi-

\footnotetext{
1 The values listed in Table 1 for BP Tau were derived before the MaPP program began using an experimental version of the magnetic imaging code. A preliminary reanalysis of this older BP Tau data using a more mature version of the code (to presented alongside newly obtained data and new magnetic maps) suggests that $B_{\text {dip }}=1.3 \mathrm{kG}, \beta_{d i p}=5^{\circ}$, $\Phi_{\text {dip }}=0.85, B_{\text {oct }}=1.8 \mathrm{kG}, \beta_{\text {oct }}=5^{\circ}$ and $\Phi_{\text {oct }}=0.15$ are more appropriate for this star in February 2006. As the new data has not yet been published we have used the old values and magnetic map for BP Tau in this paper.
}

sphere of the star, and then list the polar strength of that component as negative. For example, using this alternative approach for AA Tau then $B_{d i p}=-1.7 \mathrm{kG}, \beta_{d i p}=10^{\circ}$ and $\Phi_{d i p}=0.15$ (since tilting the negative pole of the dipole by $10^{\circ}$ towards phase 0.15 is equivalent to tilting the positive pole by $170^{\circ}$ towards phase $\left.0.15+0.5=0.65\right)$. Conservative estimates of the uncertainties in the tilts $(\beta)$ and the phases of the tilts $(\Phi)$ listed in Table 1 are $\sim 10^{\circ}$ and 0.1 (especially for moments with small tilts relative to the stellar rotation axis) respectively.

All four stars in Figure 1 and Table 1 have been observed at two different epochs. V2129 Oph showed significant changes in the strength of the dipole and octupole field components between observing epochs with the dipole component increasing by a factor of about three between 2005 and 2011 (from $0.3 \mathrm{kG}$ to $0.9 \mathrm{kG}$, see Donati et al 2011a). Likewise for TW Hya where the dipole component appeared to flip from roughly anti-aligned with the octupole component at one epoch, to being titled by about $45^{\circ}$ from an anti-parallel configuration at the other epoch; although as noted in Donati et al (2011b) this may be due to the poor phase coverage at one epoch and requires additional obser- 
vations to determine if such large scale topology changes are genuine and common for TW Hya. AA Tau and BP Tau showed little change in their field topology between observing epochs, save an overall quarter phase shift in the entire map of BP Tau. This was most likely due to a small error in the assumed rotation period building up over the roughly 40 stellar rotations between observing epochs. Changes in the field topology over time for both stars cannot be ruled out and further observations are required. We note that the magnetic maps of BP Tau were constructed and published using an experimental version of the magnetic imaging code for accreting $\mathrm{T}$ Tauri stars. The Zeeman-Doppler imaging process for such stars involves the use of polarization information in accretion related emission lines, and therefore differs from the magnetic imaging process as it applies to other stars which lack such proxies.

\section{Multipolar magnetospheric models}

In anticipation of the newly available data stream from the ESPaDOnS spectropolarimeter and the observationally derived magnetic maps of accreting pre-main sequence stars, Gregory et al. (2005) produced the first simulations of the magnetospheres of $\mathrm{T}$ Tauri stars with complex magnetic fields. The magnetic maps of young rapidly rotating zeroage main sequence stars were used to generate 3D field extrapolations which where then surrounded by accretion disks. Consequently Jardine et al (2006) and Gregory et al $(2006 a, b)$ demonstrated that magnetic fields with an observed degree of complexity were required to explain many observational results, from the increase in X-ray luminosity with stellar mass, to the common detection of rotationally modulated X-ray emission (Flaccomio et al 2005). Subsequently 3D MHD simulations of the star-disk interaction with non-dipolar fields were published by Long et al (2007, 2008) using dipole-quadrupole fields, by Long et al (2009) using dipole-octupole fields, and by Romanova et al (2011) and Long et al (2011) using dipole-octupole fields with polar field strengths and tilts that match those derived from Zeeman-Doppler imaging studies of the accreting T Tauri stars V2129 Oph and BP Tau. In addition to the field extrapolation models, and the MHD simulations, multipolar magnetic fields can also be handled analytically (or at least semi-analytically), which allows for greater clarity of understanding of the physical results from numerical models (Gregory et al 2010). Mohanty \& Shu (2008) have also presented a semi-analytic generalization of the Shu X-wind model which drops the dipole magnetosphere assumption.

The spectropolarimetric results, and the derived magnetic maps, provide clear motivation for constructing 3D models of the non-dipolar magnetospheres of pre-main sequence stars. In this section we provide a detailed review of two of the three approaches to handling multipolar stellar magnetic fields, namely field extrapolations from magnetic maps and analytic models (we compare these to MHD models in section (4). Although our attention is focussed on the magnetic fields of accreting pre-main sequence stars the results that we derive and discuss herein are applicable generally to the magnetosphere of any star or planet.

\subsection{Field extrapolations from magnetic maps}

Stellar magnetic maps derived from Zeeman-Doppler imaging can be used as inputs to models of the 3D large-scale magnetosphere. Numerical field extrapolations from magnetic maps have been produced for stars of various spectral type and evolutionary stage, for example, zero-age main sequence rapid rotators (e.g. Jardine et al 2002); accreting T Tauri stars (Jardine et al 2008; Gregory et al 2008); solartype post T Tauri stars (Dunstone et al 2008; Marsden et al 2011); a massive B star (Donati et al 2006b); and for the global solar magnetic field (e.g. Riley et al 2006; Ruan et al 2008). The potential field source surface method is used to produce the field extrapolations, a technique originally developed by the solar physics community (Altschuler \& Newkirk 1969; Schatten et al 1969). A potential field represents the lowest possible energy state of the stellar magnetic field. Twisting of the field line by stellar surface transport effects (surface differential rotation, meridional flow etc) leads to a departure from the potential state and allows magnetic energy to be stored in the field which can eventually be released through magnetic reconnection events that trigger flares.

The potential field source surface model has two boundary conditions. The first is that the radial field component at the stellar surface is the same as determined from the observationally derived magnetic map of the star whose magnetosphere is being modeled (the magnetic map is thus used as a direct input). The second boundary condition is that the field is purely radial $\left(B_{\theta}=B_{\phi}=0\right)$ at a spherical equipotential surface at some height above the star. This surface, of radius $R_{S}$, is known as the source surface and provides a simple method of mimicking the stellar wind plasma distorting and pulling open the closed field beyond a certain height above the star 2 The source surface thus represents the maximum extent that a closed field line loop can have, and adjustment of this boundary affects the amount of open and closed flux through the stellar surface reconstructed in the field extrapolation model. Smaller (larger) values of $R_{S}$ yield more (less) open field relative to closed field (see figure 1 of Gregory 2011).

In the solar case the source surface radius is constrained by in-situ heliospheric satellite observations to be $R_{S} \sim$ $2.5 \mathrm{R}_{\odot}$. For stars such in-situ satellite observations are not available and other indirect methods must be used to constrain the radius of the source surface. Although various ideas and suggestions have been put forward, $R_{S}$ remains

\footnotetext{
2 Potential field source surface models have been produced with nonspherical source surfaces (e.g. Schulz et al 1978). In the case of the Sun the source surface is a prolate spheroid at solar minimum with major axis along the solar rotation axis. At solar maximum the source surface is more complex but is approximately spherical once averaged over polar and azimuthal angles (Riley et al 2006).
} 
a poorly constrained parameter for stars other than the Sun. However, once a value for $R_{S}$ has been chosen, a coronal $\mathrm{X}$-ray emission model can be used to calculate the global $\mathrm{X}$-ray emission measure for comparison with independently obtained X-ray observations (Jardine et al 2006; Hussain et al 2007). Adjustments in $R_{S}$ affect the amount of closed field, and consequently the amount of X-ray bright regions on the star. However, once $R_{S}$ exceeds a few stellar radii the change in the calculated X-ray emission measure (or equivalently the X-ray luminosity) is small for successively larger $R_{S}$ values (Jardine et al 2008). For accreting T Tauri stars surface hot spots, which form at the shocks at the base of accretion columns, can be used as an additonal constraint on $R_{S}$. Maps of the excess emission in certain accretion related emission lines are constructed as part of the ZeemanDoppler imaging process (see Donati et al 2010b). Current observations suggest that for the majority of accreting $\mathrm{T}$ Tauri stars hot spots are at high latitude towards the visible rotation pole (see also Petrov et al 2011). In order to explain how material can accrete from the disk, in the stellar midplane, into high latitude hot spots the source surface must be large enough to ensure that closed field can reach towards the polar regions.

Potential fields are current free and therefore at every point exterior to the star $\nabla \times \mathbf{B}=\mathbf{0}$. Assuming that the field external to the star can be written as the gradient of a magnetostatic scalar potential $\Psi, \mathbf{B}=-\nabla \Psi$, then this combined with Maxwell's equation that the field is solenoidal $(\nabla \cdot \mathbf{B}=0)$ means that the magnetostatic potential at any point $(r, \theta, \phi)$ exterior to the star can be determined by solving Laplace's equation $\nabla^{2} \Psi=0$. Thus the spherical field components (e.g. $B_{r}=-\partial \Psi / \partial r$ etc) can be calculated at each point exterior to the star, and then a field line tracing algorithm employed to reconstruct the 3D global magnetic field of the star. Jardine et al (2002) provide further details of the potential field source surface method as applied to stellar magnetic maps, with Jardine et al (2006) and Gregory et al $(2006 \mathrm{a}, 2008)$ providing details of its application specifically to $\mathrm{T}$ Tauri stars. Potential field models produce unique 3D field topologies (Aly 1987), once a source surface radius has been selected, however the fields are static in time. Such models cannot be used to model the time evolution of the magnetic field which will evolve due to motion of the foot points on the stellar surface (surface transport effects) and by the twisting of the large field due to the interaction with the disk. We return to these points in section 4.

Figure 3 shows potential field extrapolations from the observationally derived magnetic map of the accreting $\mathrm{T}$ Tauri star V2247 Oph (Donati et al 2010a). This star has a complex magnetic field (see the map in Figure 2) with a weak dipole component $(<0.1 \mathrm{kG})$; none-the-less the dipole component dominates on the large scale (but see section 3.2.2). A source surface of $R_{S} \sim 4.3 R_{*}$, where $R_{*}=$ $1.6 \mathrm{R}_{\odot}$, comparable to the equatorial corotation radius for V2247 Oph $\left(R_{c o}=4.25 R_{*}\right.$ with $M_{*}=0.36 \mathrm{M}_{\odot}, P_{r o t}=$
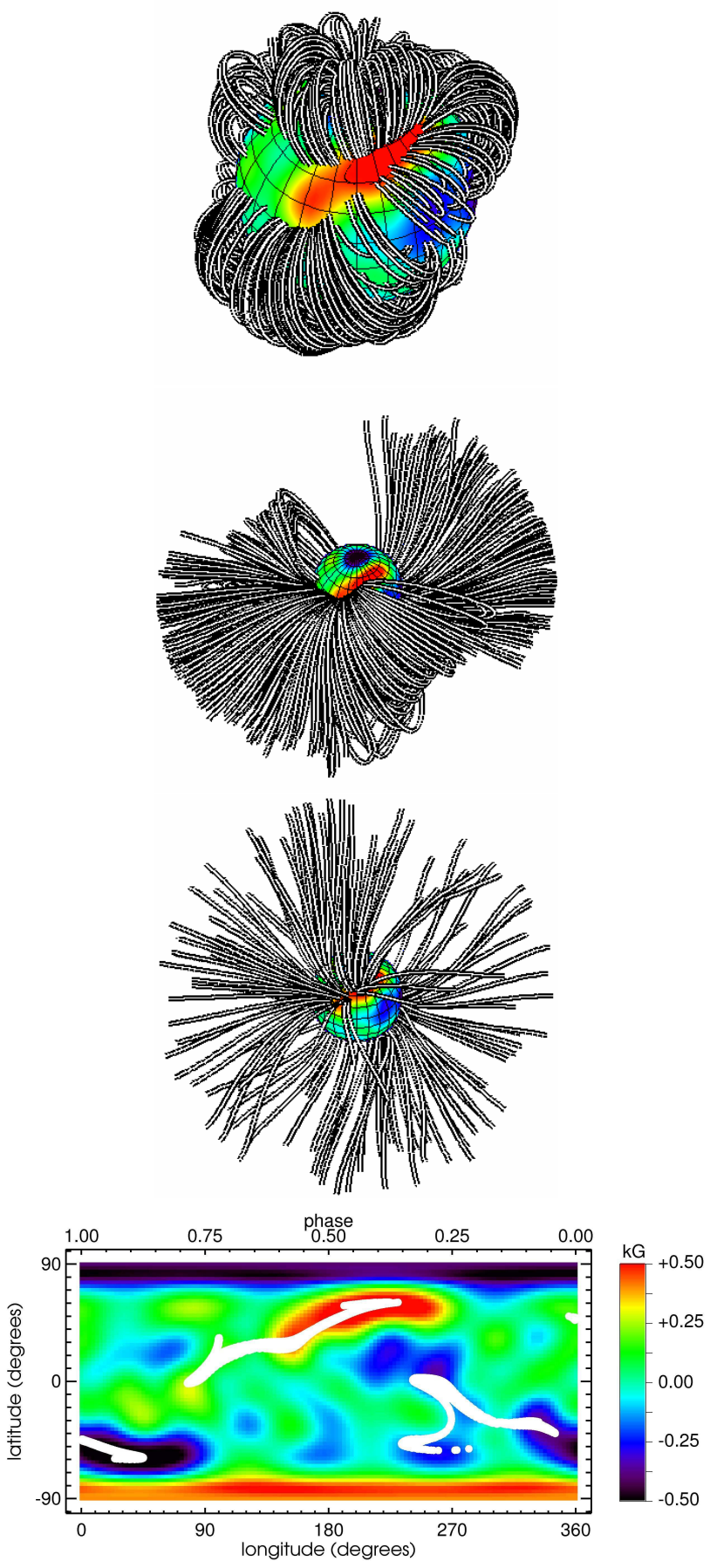

Fig. 3 Field extrapolations from the magnetic map of V2247 Oph, shown in Figure 2 and the bottom row, showing the smaller scale field that would contain high temperature plasma that constitutes the star's X-ray bright corona (top row), the larger scale field that interacts with the inner disk funneling gas on to the stellar surface (second row), and open field lines along which a stellar wind could be launched (third row). The star is viewed at inclination $45^{\circ}$ and at phase 0.5 . The top image has been expanded for clarity and is not on the same scale as the others. The white points in the plot of latitude versus longitude are the footpoints of the accretion funnels showing where the hot spots would be on the stellar surface, assuming an anti-symmetric field distribution with respect to the stellar midplane (see section 3.2.1. www.an-journal.org 
$3.5 \mathrm{~d}$ ) has been chosen for illustrative purposes. The radius is different from that in Donati et al (2010a) as we have updated the distance estimate to V2247 Oph from $140 \mathrm{pc}$ to $120 \mathrm{pc}$ (Loinard et al 2008), which yields a different luminosity and consequently a different radius. Assuming accretion occurs from a range of radii within corotation the bottom row of Figure 3 shows the location of the accretion spots on the stellar surface. The main spot in the visible hemisphere is arc-shaped and decreases in latitude towards the stellar equator from phase $\sim 0.4$ to $\sim 0.8$. This is in excellent agreement with the accretion spot location inferred from the excess emission map derived by Donati et al (2010a; see their figure 7).

\subsection{Analytic models}

Multipolar magnetic fields can also be handled analytically, including highly complex magnetic fields with many high order components. For the analysis in this work we limit ourselves to the zonal (also called axial) multipoles of arbitrary $\ell$ number (or linear combinations of $\ell$ numbers). Thus we are neglecting the non-axisymmetric field modes (those with $m \neq 0$ ). The same assumption is made in the generalized multipolar X-wind model of Mohanty \& Shu (2008) and in the initial field structures used as inputs in current 3D MHD simulations (Romanova et al 2011; Long et al 2011; see section (4). Observationally the magnetic fields of accreting $\mathrm{T}$ Tauri stars are dominantly axisymmetric with the exception of some low mass stars (typically below $0.5 \mathrm{M}_{\odot}$ ) and older/more massive $\mathrm{T}$ Tauri stars with substantial radiative cores (those with $M_{\text {core }} / M_{*} \geq 0.4$; Gregory et al 2012).

Gregory et al (2010) derived an expression for the spherical magnetic field components $\left(B_{r}, B_{\theta}, B_{\phi}\right)$ exterior to a star for a multipole of order $\ell$ (where $\ell=1,2,3,4, \ldots$ are the dipole, the quadrupole, the octupole, the hexadecapole etc),

$$
\begin{aligned}
\mathbf{B}= & \frac{B_{*}^{\ell, p o l e}}{(\ell+1)}\left(\frac{R_{*}}{r}\right)^{\ell+2} \times \\
& \sum_{k=0}^{N}\left\{\frac{(-1)^{k}(2 \ell-2 k) !}{2^{\ell} k !(\ell-k) !(\ell-2 k) !}(\hat{\boldsymbol{\mu}} \cdot \hat{\mathbf{r}})^{\ell-2 k} \times\right. \\
& {\left.\left[(2 \ell-2 k+1) \hat{\mathbf{r}}-(\ell-2 k)(\hat{\boldsymbol{\mu}} \cdot \hat{\mathbf{r}})^{-1} \hat{\boldsymbol{\mu}}\right]\right\}, }
\end{aligned}
$$

where $N=\ell / 2$ or $(\ell-1) / 2$ whichever is an integer, $B_{*}^{\ell, p o l e}$ is the polar field strength of the $\ell$-number multipole, $\hat{\mathbf{r}}$ is a unit vector along the radial direction and $\hat{\mu}$ is a unit vector in the direction of the multipole moment $\mu$. Magnetic fields consisting of multipoles with many high order $\ell$-number components can be constructed by summing equation (1) over the $\ell$ numbers of interest.

In the following subsections we derive expressions for the spherical field components that are written in terms of the tilt and phase of tilt of each multipole moment explicitly. Using these expressions we construct simulated magnetic maps for the four stars shown in Figure 1, all of which have large scale fields that are well described by tilted dipole plus tilted octupole components. We compare the simulated maps to the maps derived from Zeeman-Doppler imaging which contain information about many higher order $\ell$ number and $m \neq 0$ field modes. We further derive generalized expressions for the stellar disk averaged longitudinal field component and demonstrate that reliance on this sole diagnostic provides poor constrains on the overall surface magnetic field topology (e.g. Donati \& Landstreet 2009).

\subsubsection{Tilted multipolar magnetic fields}

We consider an axial multipole ( $m=0$ ) of order $\ell$ tilted by an angle $\beta$ relative to the stellar rotation axis and tilted towards a longitude $\psi$ where $\psi$ is related to the rotation phase $\Phi$ via

$$
\psi=(1-\Phi) \times 360^{\circ} .
$$

Note that rotation phase $\Phi$ and longitude $\psi$ run backwards such that longitudes $\psi=0^{\circ}, 90^{\circ}, 180^{\circ}, 270^{\circ}$ and $360^{\circ}$ correspond to rotation phases $\Phi=1,0.75,0.5,0.25$ and zero, and that our derivation assumes the $x z$-plane corresponds to a rotation phase of zero (see Appendix A). The spherical field components can be derived from equation (1), following the details of Appendix $\mathrm{A}$ and by noting that as $\hat{\boldsymbol{\mu}}=\mu^{-1}\left(\mu_{r}, \mu_{\theta}, \mu_{\phi}\right)$ then $\hat{\boldsymbol{\mu}} \cdot \hat{\mathbf{r}}=\mu_{r} / \mu$,

$$
\begin{aligned}
& B_{r}=B_{*}^{\ell, \text { pole }}\left(\frac{R_{*}}{r}\right)^{\ell+2} \times \\
& \sum_{k=0}^{N}\left\{\frac{(-1)^{k}(2 \ell-2 k) !}{2^{\ell} k !(\ell-k) !(\ell-2 k) !}\left(\frac{\mu_{r}}{\mu}\right)^{\ell-2 k}\right\} \\
& B_{\theta}=\frac{B_{*}^{\ell, \text { pole }}}{(\ell+1)}\left(\frac{R_{*}}{r}\right)^{\ell+2} \times \\
& \sum_{k=0}^{N}\left\{\frac{(-1)^{k+1}(2 \ell-2 k) !}{2^{\ell} k !(\ell-k) !(\ell-2 k-1) !}\left(\frac{\mu_{r}}{\mu}\right)^{\ell-2 k-1} \frac{\mu_{\theta}}{\mu}\right\} \\
& B_{\phi}=\frac{B_{*}^{\ell, p o l e}}{(\ell+1)}\left(\frac{R_{*}}{r}\right)^{\ell+2} \times \\
& \sum_{k=0}^{N}\left\{\frac{(-1)^{k+1}(2 \ell-2 k) !}{2^{\ell} k !(\ell-k) !(\ell-2 k-1) !}\left(\frac{\mu_{r}}{\mu}\right)^{\ell-2 k-1} \frac{\mu_{\phi}}{\mu}\right\} .
\end{aligned}
$$

where we again note that $N=\ell / 2$ or $(\ell-1) / 2$ whichever is an integer, $\mu=|\boldsymbol{\mu}|$, and the components of the multipole moment $\boldsymbol{\mu}$ are

$$
\begin{aligned}
\mu_{r} & =\mu \sin \theta \cos \phi \cos \psi \sin \beta+\mu \sin \theta \sin \phi \sin \psi \sin \beta \\
& +\mu \cos \theta \cos \beta \\
\mu_{\theta} & =\mu \cos \theta \cos \phi \cos \psi \sin \beta+\mu \cos \theta \sin \phi \sin \psi \sin \beta \\
& -\mu \sin \theta \cos \beta \\
\mu_{\phi} & =-\mu \sin \phi \cos \psi \sin \beta+\mu \cos \phi \sin \psi \sin \beta
\end{aligned}
$$

where $\theta$ and $\phi$ are standard spherical coordinates in the reference frame of the star. The components of a multipolar magnetosphere can then be determined by summing the appropriate field components. For example, a dipole plus octupole magnetosphere where the dipole (octupole) moment 
of polar strength $B_{d i p}\left(B_{o c t}\right)$ is titled by $\beta_{d i p}\left(\beta_{\text {oct }}\right)$, relative to the stellar rotation axis, towards a rotation phase denoted by $\psi_{d i p}\left(\psi_{o c t}\right)$ has spherical components

$$
\begin{aligned}
B_{r} & =B_{r}\left(B_{d i p}, \beta_{d i p}, \psi_{d i p}, \ell=1\right) \\
& +B_{r}\left(B_{o c t}, \beta_{o c t}, \psi_{o c t}, \ell=3\right) \\
B_{\theta} & =B_{\theta}\left(B_{d i p}, \beta_{d i p}, \psi_{d i p}, \ell=1\right) \\
& +B_{\theta}\left(B_{o c t}, \beta_{o c t}, \psi_{o c t}, \ell=3\right) \\
B_{\phi} & =B_{\phi}\left(B_{d i p}, \beta_{d i p}, \psi_{d i p}, \ell=1\right) \\
& +B_{\phi}\left(B_{o c t}, \beta_{o c t}, \psi_{o c t}, \ell=3\right)
\end{aligned}
$$

where $B_{d i p} \equiv B_{*}^{1, \text { pole }}$ and $B_{\text {oct }} \equiv B_{*}^{3, \text { pole }}$.

We note that the field components derived in this section for tilted axial multipoles (those with $m=0$ ) also apply to sectoral multipoles (those with $\ell=m$ ) which are axial (zonal) multipoles with the moment symmetry axis tilted into the equatorial plane, i.e. perpendicular to the stellar rotation axis.

As a test of the above Figure 4 shows simulated magnetic surface maps for the four stars shown in Figure 1 The polar strength of the dipole and octupole components, the phase they are tilted towards, and their tilts relative to the stellar rotation axis match those listed in Table 1. For comparison we also show in Figure 4 the observationally derived magnetic surface maps in the same Cartesian (i.e. latitude vs longitude) grid format. The $\ell$ number of a multipole determines the number of polarity changes in the surface field if one moves along a line of constant longitude from the north to the south pole of the star (or vice versa). The domination of the dipole component $(\ell=1)$ in the AA Tau map and the octupole component $(\ell=3)$ in the TW Hya map is clear.

In order to produce field extrapolations some assumption has to be made about the form of the magnetic field in the $\sim$ hemisphere of the star that always remains hidden from view to an observer. In Figure 4 we have assumed that the field is dominantly antisymmetric with respect to the stellar midplane, and thus the odd $\ell$ number modes dominate. If we assume that the even $\ell$ number modes dominate then the resulting large scale field topologies would result in magnetospheric accretion occurring primarily on to lower, equatorial, latitudes. As this is typically not observed, and in order to ensure that material from the disk is able to accrete into the high latitude hot spots that are observed, we favor the antisymmetric field modes and assign the field in the hidden hemisphere accordingly. We note that this assumption has been examined in detail by Johnstone et al (2010) who conclude that for accreting $\mathrm{T}$ Tauri stars, the field in the visible hemisphere that is reconstructed in the magnetic maps remains largely unaffected by the assumptions of the form of the magnetic field in the hidden hemisphere.

The effect of higher order field modes is apparent in the observationally derived surface magnetic maps shown in Figure 4, particularly for V2129 Oph and BP Tau. The high latitude single polarity magnetic spots are stronger and of different shape in the observationally derived maps compared to the simulated maps constructed from dipole and octupole field components only. This is caused by the presence

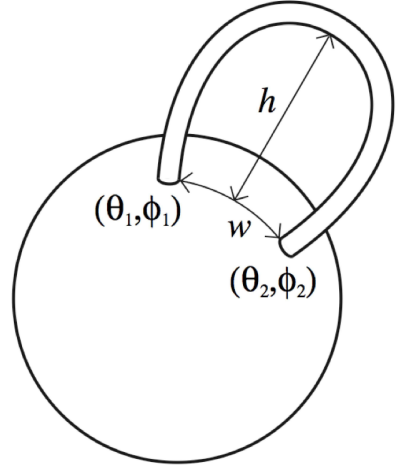

Fig. 5 Cartoon illustrating the definition of the height $h$ and the width $w$ of a closed field line loop with foot points at colatitudes and longitudes $\left(\theta_{1}, \phi_{1}\right)$ and $\left(\theta_{2}, \phi_{2}\right)$.

of higher order $\ell$ numbers in the observed maps. Likewise there is more azimuthal structure apparent in the observed maps; in particular within the mid-latitude negative field band on both V2129 Oph and BP Tau. This is caused by the presence of non-axisymmetric field components (those with $m \neq 0$ ) in the observed maps (further details can be found in the papers where the magnetic maps have been published; Donati et al 2011a for V2129 Oph; Donati et al 2008 for BP Tau; Donati et al 2010b for AA Tau; and Donati et al 2011b for TW Hya). The maps derived from ZeemanDoppler imaging thus contain more information than can be captured by the simple model consisting of tilted dipole plus tilted octupole field components only. None-the-less it is apparent from Figure 4 that the simple dipole-octpole model provides excellent overall agreement on their large scale field topologies; it is such dipole-octupole models that have been used as the input conditions in the latest generation of 3D MHD simulations of the star-disk interaction (Romanova et al 2011; Long et al 2011). In the following section we compare the large scale field topology derived via field extrapolation from the two sets of surface maps presented in Figure 4

\subsubsection{Comparison with field extrapolations}

We have carried out field extrapolations from the magnetic surface maps derived from Zeeman-Doppler imaging and those constructed assuming only tilted dipole and tilted octupole field components shown in Figure 4 This allows for a quantitative comparison between the 3D magnetospheric topologies derived from both sets of maps. To compare the field topologies we calculate the height and width of the closed field line loops, as defined in Figure 5. The height $h$ is the maximum height the loop reaches above the stellar surface, and the width $w$ is the distance between the loop foot points as measured along the segment of the great circle that passes through the foot points on the stellar surface. Details of how these quantities are calculated can be found in Gregory et al (2008). 

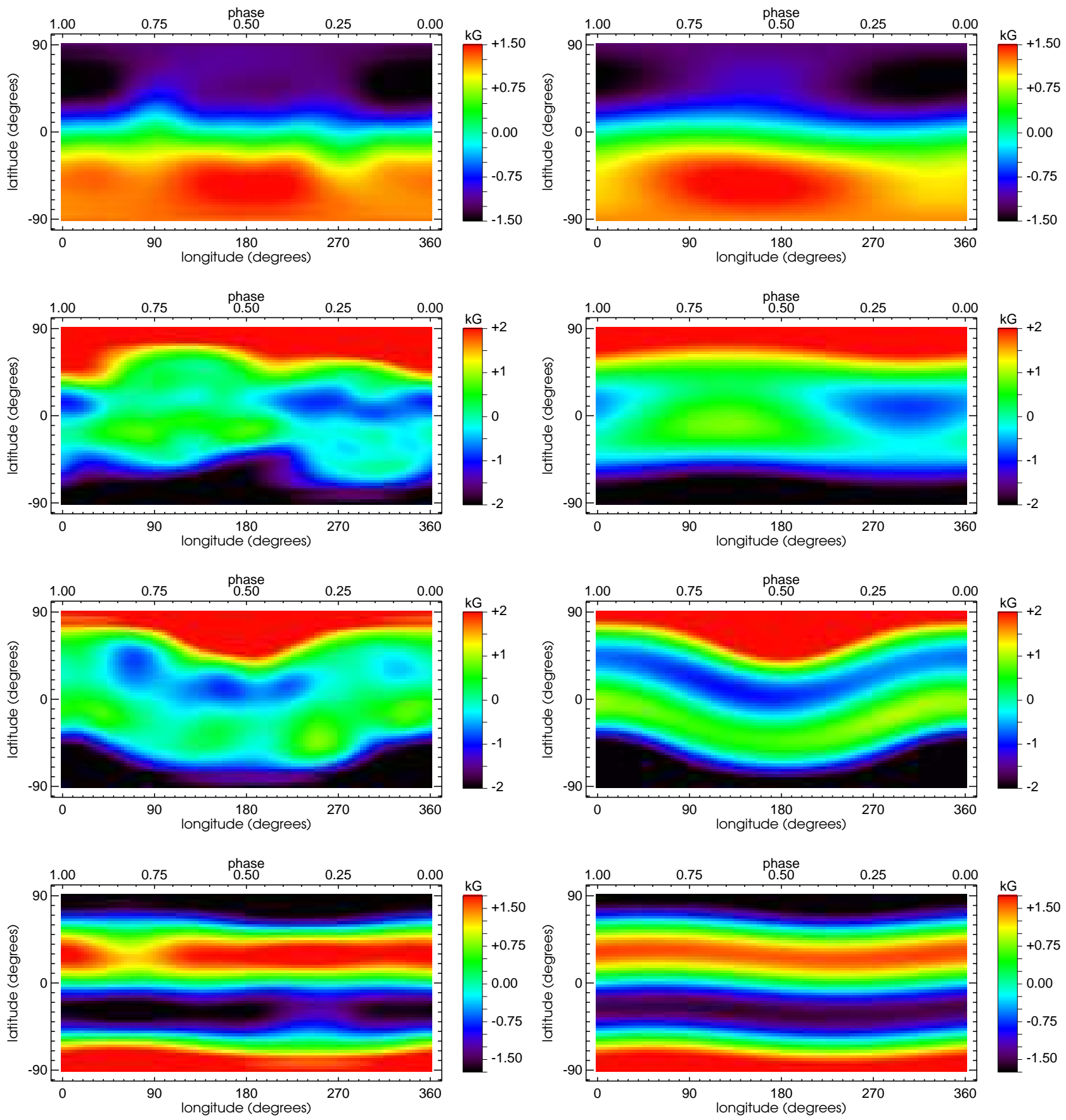

Fig. 4 (left) Observationally derived magnetic maps assuming antisymmetric field configurations with respect to the stellar midplane for (top to bottom) AA Tau in January 2009, BP Tau in February 2006, V2129 Oph in July 2009 and TW Hya in March 2010. (right) simulated magnetic maps constructed using tilted dipole and tilted octupole components with the same polar field strength, tilts, and phases of tilt as determined from the observationally derived magnetic maps as listed in Table 1 
Figure 6 shows the comparison between the field extrapolations from the maps in Figure 4. For simplicity we have set the source surface to be at approximately the equatorial corotation radius for each star. We remind readers that the source surface denotes the maximum extent of the closed field, and thus a field line cannot have a height greater than the radius of the source surface; in this case corotation. Larger scale field lines are likely torn open due to the interaction with the disk (e.g. Matt \& Pudritz 2005), as has also been found in MHD simulations (e.g. Romanova et al 2011). The maximum width that a field line could have is less than $\pi R_{*}$ (loops with a larger width would be wider than the star, which is clearly unphysical). On each plot in Figure 6 the solid red line shows the relationship between $h$ and $w$ for a pure dipole field, and the solid green lines for a pure octupole field. There are two such lines for the octupole case as for an axisymmetric octupole there are three rings of closed field about the star, and the higher latitude rings are of different width compared to the equatorial ring (this is due to the mathematical properties of the associated Legendre functions, see Gregory 2011).

It is clear from Figure 6 that there is good agreement between the 3D field structures obtained via field extrapolation from the observationally derived (left column), and the simulated dipole-octupole (right column), magnetic maps. Better agreement is however obtained for AA Tau (top row) and TW Hya (bottom row). These are the stars which have one of the dipole or octupole components clearly dominant over the other; AA Tau hosting a dominantly dipolar magnetic field, and TW Hya a dominantly octupolar one (see Table 1). The agreement is poorer, although not significantly so, for BP Tau and V2129 Oph (second and third rows of Figure 6), stars which have both strong dipole and octupole field components; although for V2129 Oph/BP Tau the octupole/dipole component contains the bulk of the magnetic energy (Donati et al 2011a, 2008). For V2129 Oph in particular there are many field lines with large widths $\left(\sim 2 R_{*}\right)$ but small heights $\left(\sim 1 R_{*}\right)$ that are apparent in the field extrapolation from the observationally derived map (see Figure 4 first column third row) but which are missing from the extrapolation from the simulated map containing the tilted dipole and tilted octupole field components only (see Figure 4 second column third row). These field lines connect the strong negative field regions evident in the mid-latitude northern hemisphere to equivalent positive field regions in the other hemisphere. This azimuthal field structure which arises due to the presence of non-axisymmetric $(m \neq 0)$ field modes can be seen in the observationally derived map. As the simulated maps neglect the $m \neq 0$ field modes for simplicity, a group of field lines is missed in the field extrapolation from the V2129 Oph simulated map, which explains the difference seen in Figure 6 Given that these field lines are limited in height their presence does not significantly affect the structure of the larger scale field which is interacting with the disk, and is thus unlikely to have any effect on the results of MHD modeling which use dipole-octupole fields as inputs (see section 4).

AA Tau and TW Hya have field configurations where the dipole and octupole components are close to an antiparallel configuration, whereas for BP Tau and V2129 Oph they are close to a parallel configuration (at least at the epochs listed in Table 1). The differing field configurations are clearly apparent in the global field structure and in the plots of field line height versus width, see Figure 6 The largest field lines, those with the greatest height, for AA Tau and TW Hya have smaller width at the same height compared to a pure dipole, i.e. the black points in Figure 6 for these stars lie to the left of the red line that represents the dipole. The opposite is the case for BP Tau and V2129 Oph where the field lines with the greatest height are wider than those of a dipole (as already discussed in Gregory et al 2008) i.e. the black points in Figure 6lie to the right of the red line.

In all cases the magnitude of this effect, and the departure from a dipole field, is greater for larger values of the ratio of the polar field strength of the octupole to the dipole component $B_{\text {oct }} / B_{\text {dip }}$. This can be understood as follows. The dipole and octupole components for each of the four stars listed in Table 1 are tilted by different amounts towards different rotation phases. However, as the tilts are small, let's assume that V2129 Oph and BP Tau have dipoleoctupole fields where the moments are both aligned with the stellar rotation axis and parallel to one another, $\beta_{\text {dip }}=$ $\beta_{\text {oct }}=0^{\circ}$ (main positive poles coinciding with the visible rotation pole). Likewise, let's assume that AA Tau and TW Hya have dipole-octupole fields where the moments are both aligned with the stellar rotation axis but are antiparallel to one another, $\beta_{d i p}=0^{\circ}, \beta_{\text {oct }}=180^{\circ}$ (positive/main negative pole of the dipole/octupole coinciding with the visible rotation pole; TW Hya is close to this configuration) or $\beta_{\text {dip }}=180^{\circ}, \beta_{\text {oct }}=0^{\circ}$ (negative/main positive pole of the dipole/octupole coinciding with the visible rotation pole; AA Tau is close to this configuration). The field components for the simplified cases of aligned and anti-aligned dipole-octupole fields can be derived from equations (3) 5) using (6) 8 ),

$$
\begin{aligned}
& B_{r}=B_{d i p}\left(\frac{R_{*}}{r}\right)^{3} \cos \theta \cos \beta_{d i p}+ \\
& \frac{1}{2} B_{o c t}\left(\frac{R_{*}}{r}\right)^{5}\left(5 \cos ^{2} \theta \cos ^{2} \beta_{o c t}-3\right) \cos \theta \cos \beta_{o c t} \\
& B_{\theta}=\frac{1}{2} B_{d i p}\left(\frac{R_{*}}{r}\right)^{3} \sin \theta \cos \beta_{d i p}+ \\
& \frac{3}{8} B_{o c t}\left(\frac{R_{*}}{r}\right)^{5}\left(5 \cos ^{2} \theta \cos ^{2} \beta_{o c t}-1\right) \sin \theta \cos \beta_{o c t}
\end{aligned}
$$

where $B_{\phi}=0$ as the fields under consideration are axisymmetric, and the first/second terms in each component are the contributions from the dipole/octupole part of the field. Using these field components we can solve the differ- 

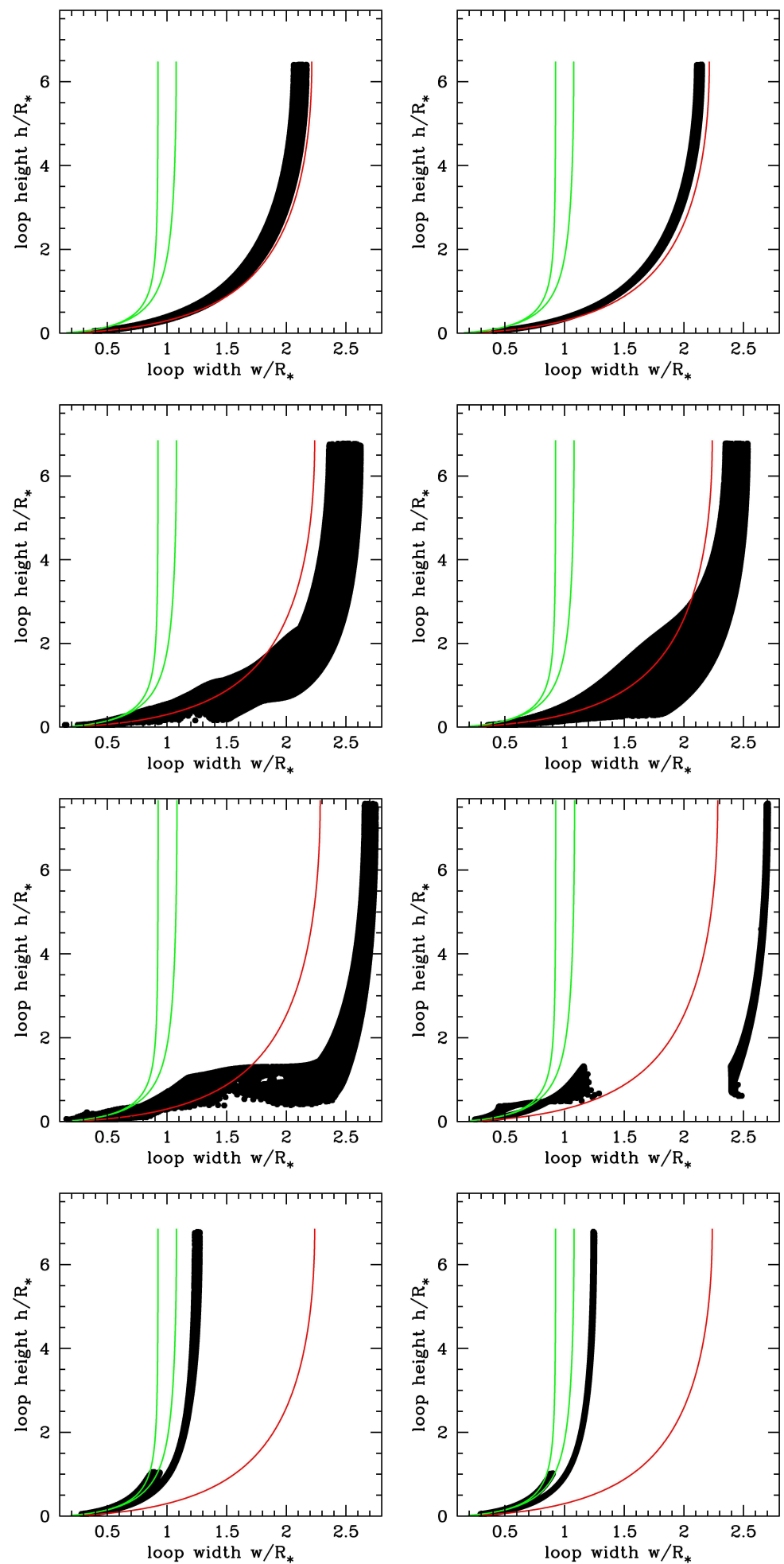

Fig. 6 Height $h$ versus width $w$ plots for the field lines obtained via field extrapolation from the magnetic maps in Figure 4 (black points). Plots corresponding to the observationally derived maps are shown on the left, and those from the simulated maps assuming only tilted dipole plus tilted octupole field components are shown on the right for, from top to bottom, AA Tau, BP Tau, V2129 Oph and TW Hya. On each plot the red line shows the height-width relation for an axial dipole field $(\ell=1, m=0)$, and the green lines the equivalent for an axial octupole field $(\ell=3, m=0)$. 
ential equation describing the path of the field lines,

$$
\frac{B_{r}}{\mathrm{~d} r}=\frac{B_{\theta}}{r \mathrm{~d} \theta},
$$

and determine the structure of the magnetic field external to the star. By path of the field lines we mean a function of the form $r=r(\theta)$ that describes the field lines in spherical coordinates. For example, for an aligned dipole (set $B_{\text {oct }}=$ 0 and $\beta_{d i p}=0^{\circ}$ in equations (12) and (13)) the result is $r=$ const. $\sin ^{2} \theta$ where the constant is the maximum radial extent of the dipole loops in the stellar midplane (a result commonly found in the literature e.g. Gregory 2011).

For the dipole-octupole fields considered here, and in fact for any arbitrary combination of axial $\ell$ number multipoles, equation (14) has an analytic solution; however, the mathematics is just too miserable to detail here. Equation (14), with equations (12) and (13), can also be solved numerically. Figure 7 shows the axisymmetric dipole-octupole magnetic fields for two cases (i) for dipole and octupole components that are aligned with the rotation axis and parallel to one another with $\beta_{d i p}=\beta_{o c t}=0^{\circ}$ (a configuration similar to that of V2129 Oph and BP Tau), with $B_{o c t} / B_{d i p}=4.0$ to more clearly emphasize the difference between this field and a dipole; and (ii) for dipole and octupole components that are aligned with the rotation axis and anti-parallel with $\beta_{d i p}=0^{\circ}$ and $\beta_{o c t}=180^{\circ}$ (a configuration similar to that of TW Hya) again with $B_{\text {oct }} / B_{\text {dip }}=$ 4.0. On each plot the red lines show some dipole field lines passing through the stellar midplane at the same position as some of the dipole-octupole field lines (black lines). The field topology depends only on the ratio $B_{o c t} / B_{d i p}$ rather than on the polar strengths of the individual field components.

The field line plots in Figure 7 provide an immediate explanation for the differences found between the extrapolated fields (the black points) and the dipole fields (red lines) in Figure 6 For stars like V2129 Oph and BP Tau where the dipole and octupole moments are roughly parallel, the large scale field lines are squeezed by the octupole field line loops as we move along the large scale field lines from the stellar midplane towards the stellar surface. This can seen in Figure 7 (top panel) by comparing the dipoleoctupole (black) and the dipole (red) field lines. In such cases the large scale field lines of the dipole-octupole fields have larger width at the stellar surface than dipole field lines of the same height due to the squeezing by the more complex surface field regions. This effect becomes greater the larger the ratio of $B_{o c t} / B_{d i p}$, i.e. the greater the influence of the octupole over the dipole component. This be seen in Figure 8 where, for a field line of a fixed height, we compare the width of the dipole-octupole field line relative to that of a dipole field line as we vary the ratio $B_{\text {oct }} / B_{\text {dip }}$. As V2129 Oph has a larger $B_{o c t} / B_{d i p}$ ratio than BP Tau this squeezing effect is greater for V2129 Oph, and thus the large scale field lines of a given height are even wider than those of a dipole for V2129 Oph than they are for BP Tau (see Figure 6 second and third rows and Figure 8). For field
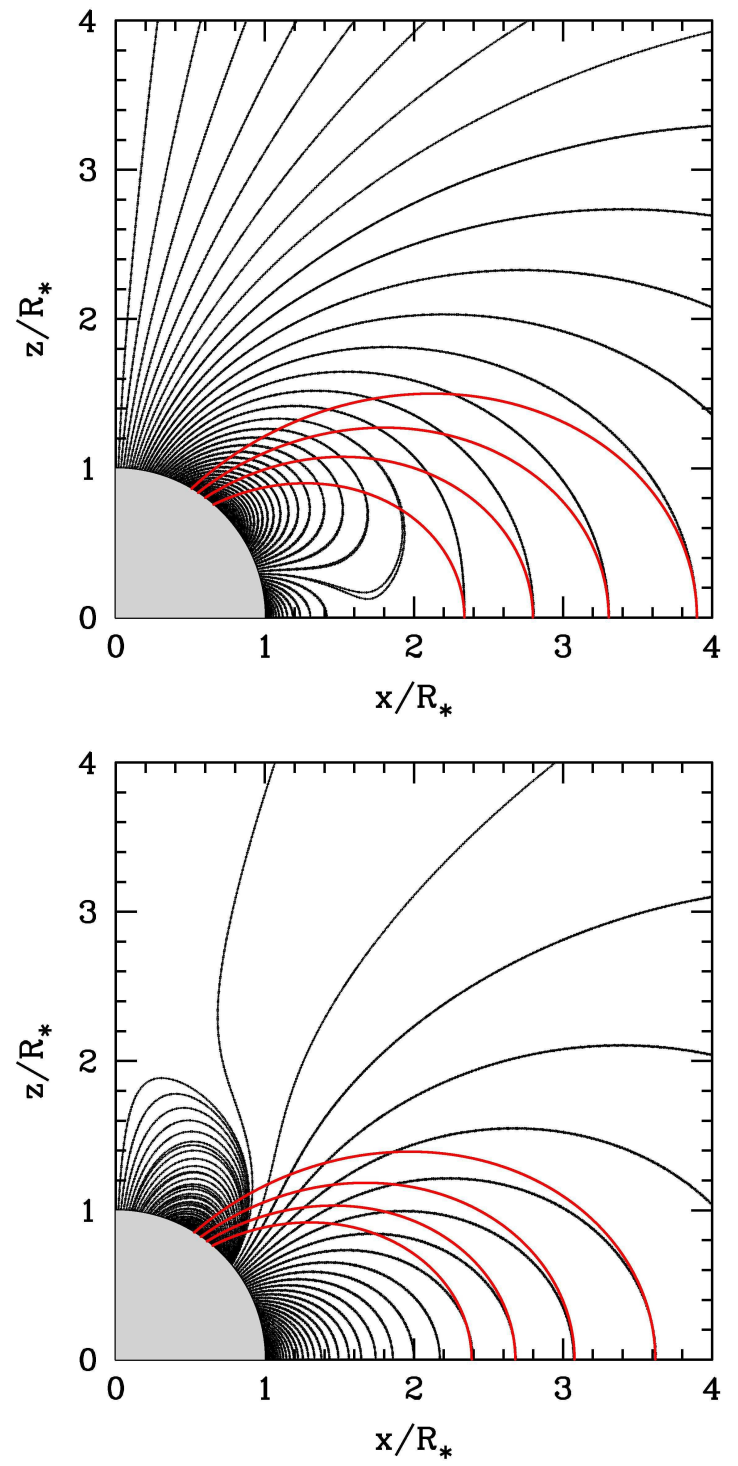

Fig. 7 Field lines (black) for an axisymmetric dipoleoctupole field where the dipole and octupole components are parallel with $\beta_{d i p}=\beta_{o c t}=0^{\circ}$ (top panel), and where the dipole and octupole components are anti-parallel with $\beta_{d i p}=0^{\circ}$ and $\beta_{o c t}=180^{\circ}$ (bottom panel). $B_{\text {oct }} / B_{\text {dip }}=$ 4.0 in both cases. For comparison some dipole field lines are shown in red. The larger scale dipole-octupole field lines are squeezed by the more complex surface field. As the field lines are rotationally symmetric about the $z$-axis and reflectionally symmetric in the $x$-axis only one quadrant is shown.

topologies like AA Tau and TW Hya, where the dipole and octupole moments are close to anti-parallel, the large scale field lines are distorted in a different way close to the stellar surface, see Figure 7 (bottom panel). In such cases the large scale field lines have smaller width at the stellar surface than dipole field lines of the same height, explaining the difference found in Figure 6 for AA Tau and TW Hya (first and fourth rows) between the extrapolated and dipole fields. As TW Hya has the largest $B_{o c t} / B_{d i p}$ value the ef- 


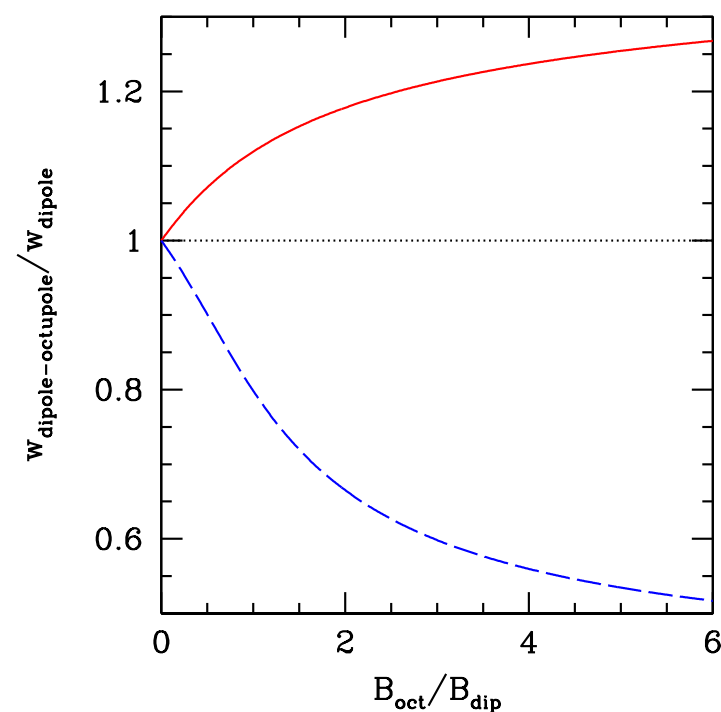

Fig. 8 The variation in the ratio of the width of a large closed field line loop for an axisymmetric dipole-octupole magnetic field $w_{\text {dipole-octupole }}$ relative to the width of a dipole loop $w_{\text {dipole }}$ of the same height as a function of the ratio of the polar field strength of the octupole to the dipole $B_{\text {oct }} / B_{\text {dip }}$. For comparative purposes a height of $h=4 R_{*}$ has been chosen, which corresponds to a field line threading a disk at a radius of $5 R_{*}$, a typical disk truncation radius. The width and height are defined in Figure 5. The solid red line shows the case where the dipole and octupole components are parallel; in this senario, relevant to BP Tau and V2129 Oph, the large scale field lines are wider than the pure dipole case. The dashed blue line shows the case where the dipole and octupole and anti-parallel; in this senario, relevant to AA Tau and TW Hya, the large scale field lines are of smaller width than the pure dipole case.

fect is greatest for this star, but is (almost) negligible for the dominantly dipolar magnetic field of AA Tau (the field line plot using the AA Tau $B_{\text {oct }} / B_{\text {dip }}$ ratio shows very little difference from a pure dipole). The results of this section demonstrate how an analytic model can be used to further our understanding of the results obtained from numerical (field extrapolation) models.

The effect of the large scale field structure being distorted close to the stellar surface by the complex surface field regions is important for models of accretion flow along the field lines on to the stellar surface (Adams \& Gregory 2011). Not only is the hot spot location a sensitive function of the magnetic field topology (e.g. Gregory et al 2005, 2006a; Mohanty \& Shu 2008), but the pre-shock density of gas can be up to an order of magnitude larger than that predicted from dipolar accretion models (Gregory et al 2007). This is straightforward to understand physically if we assume that accretion occurs from the stellar midplane (e.g. imagine a flat disk in the equatorial plane of Figure 7). Assuming mass conservation then the mass flux through the cross sectional area $A$ of the accretion column is constant, i.e. $\rho v A=$ const. where $\rho$ and $v$ are the flow density and velocity, and the constant is the mass accretion rate. The infall speed for gas coupled to the magnetic field is roughly the same for both accretion along dipole, and non-dipolar field lines, since the infall velocity is essentially free-fall and determined by the depth of the gravitational potential well of the star. However, the cross-sectional area of the dipole-octupole field lines becomes smaller than that of the dipole case (see Figure 7), and therefore to ensure that the mass flux remains constant, the flow density must increase by roughly the same factor that the cross sectional area reduces.

\subsubsection{Longitudinal field component}

When high-resolution Stokes V (circular polarization) data is available detailed modeling of the profiles and their rotational modulation provides the best information about stellar magnetic field topologies, and allows magnetic maps to be constructed. However, estimates of the stellar disk averaged longitudinal field component (also called the transverse or line-of-sight field component) $B_{l o n}$ and how this varies with rotation phase $\Phi$ is commonly found in the literature. The longitudinal field component is an integrated quantity over the visible disk of the star, and thus is often a poor diagnostic of the surface field topology due to the flux cancellation effect. If the stellar surface was covered in equal amounts of positive and negative field the measured stellar disk averaged longitudinal field component would be zero, yet in such cases the Stokes V profile itself may show a clear Zeeman signature (e.g. see the longitudinal field curve from the LSD average photospheric absorption line and the Stokes V profile for V2129 Oph around phase 0.78 in July 2009; Donati et al 2011a).

Large scale magnetic topologies have been probed using the oblique rotator model, where a dipole field is assumed to be tilted by an angle $\beta_{d i p}$ relative to the stellar rotation axis with a stellar inclination of $i$. The longitudinal field component thus varies with rotation phase, reaching a maximum when the star is viewed at a rotation phase $\Phi$ that corresponds to the phase that the dipole component is tilted towards $\Phi_{\text {dip }}$ (assuming it is the positive pole of the dipole in the visible hemisphere). Here we extend our analytic work by deriving an expression for the longitudinal magnetic field component for dipole-octupole magnetic fields. Even for such simple field structures, and where the octupole component is stronger than the dipole component, we demonstrate below that the phase modulated longitudinal field curve is dominated by the dipole component (although the polar field strength and tilt of this component is also poorly constrained by the longitudinal field curve once cool spots, neglected in this simple model, are accounted for - see below), and therefore provides poor constraints on the stellar field topology. Detailed modeling of rotationally modulated Zeeman signatures, when high resolution spectropolarimetric data is available, provides better and more 
detailed constraints on stellar magnetic topologies (see Donati \& Landstreet 2009 for further details on the limitations of the longitudinal magnetic field component).

Although the expression for the longitudinal field component for an inclined dipole magnetosphere is commonly found in the literature (e.g. Preston 1967), we cannot find explicit expressions for a tilted octupole magnetosphere, although we note that Bagnulo et al (1996) did use a combination of a tilted dipole, a tilted quadrupole and a tilted octupole in their work. Assuming a linear limb darkening law the stellar disk averaged longitudinal magnetic field component for a dipole-octupole field as a function of rotation phase is (see Appendix B for a derivation of this result),

$$
\begin{aligned}
& B_{\text {lon }}(\Phi)=\frac{1}{20} B_{\text {dip }}\left(\frac{15+u}{3-u}\right) \times \\
& \left(\cos \beta_{\text {dip }} \cos i+\sin \beta_{\text {dip }} \sin i \cos \left[2 \pi\left(\Phi-\Phi_{\text {dip }}\right)\right]\right) \\
& +\frac{1}{16} B_{\text {oct }}\left(\frac{u-1}{3-u}\right) \times \\
& \left\{5\left(\cos \beta_{\text {oct }} \cos i+\sin \beta_{\text {oct }} \sin i \cos \left[2 \pi\left(\Phi-\Phi_{\text {oct }}\right)\right]\right)^{3}-\right. \\
& \left.3\left(\cos \beta_{\text {oct }} \cos i+\sin \beta_{\text {oct }} \sin i \cos \left[2 \pi\left(\Phi-\Phi_{\text {oct }}\right)\right]\right)\right\},
\end{aligned}
$$

where $B_{d i p}$ is the polar strength of the dipole component, $\beta_{\text {dip }}$ the tilt of the positive pole of the dipole relative to the visible stellar rotation pole, $\Phi_{d i p}$ is the rotation phase that the dipole moment is tilted towards, with $B_{\text {oct }}, \beta_{\text {oct }}$ and $\Phi_{\text {oct }}$ the same respective quantities for the octupole field component. $\Phi$ is the rotation phase, and $u$ is the linear limb darkening coefficient. The stellar inclination is $i$, such that the observer's reference frame $\left(x_{o b s}, y_{o b s}, z_{o b s}\right)$ has $z$-axis parallel to the line of sight i.e. we can transform from the stellar reference frame $(x, y, z)$ to the observer's frame $\left(x_{o b s}, y_{o b s}, z_{o b s}\right)$, which share the stellar center as the coordinate origin, by rotating the stellar frame counterclockwise by an angle $i$ when looking down the $y$-axis towards the origin.

In Figure 9 we illustrate the longitudinal field component as a function of rotation phase for a star with a dipoleoctupole field with the same parameters as BP Tau listed in Table 1 It is clear that the longitudinal field curve is dominated by the dipole component of the magnetic field even though the octupole field component is stronger. $\mathrm{Ob}-$ servationally the octupole component may easily be missed by analyzing the longitudinal field curve alone once a limited phase coverage and errors are accounted for, despite being easily recoverable if a full analysis is made of the rotational modulation of the Stoke V signal, as is done in Zeeman-Doppler imaging and in the construction of stellar magnetic maps. The situation is worse for higher order field components. Furthermore, even though the longitudinal field curve is only weakly sensitive to the high order field components in the simple model presented here, it cannot be used as a probe of the large-scale dipole component. In this simple analytic dipole-octupole field model we have not accounted for the presence of surface cool (dark) spots.

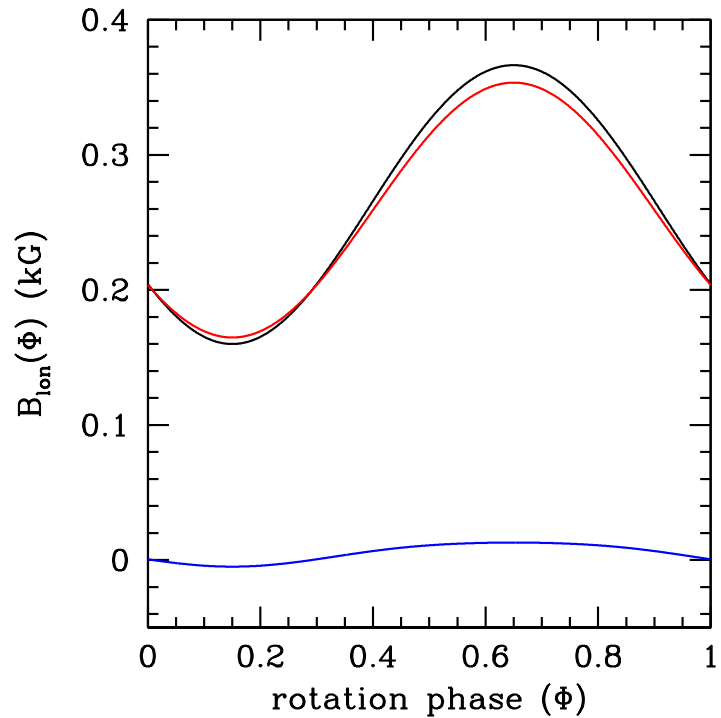

Fig.9 The stellar disk averaged longitudinal magnetic field component $B_{l o n}$ as a function of rotation phase $\Phi$ for a star with a tilted dipole plus tilted octupole magnetic field with tilts, phases of tilt, and polar field strength of the components matching those determined from the observationally derived magnetic map of BP Tau, see Table 1. $B_{l o n}$ (black line) is calculated from equation (15) assuming a linear limb darkening coefficient of $u=0.6$ and a stellar inclination of $45^{\circ}$. The red/blue line shows the contribution to $B_{l o n}$ from the dipole/octupole component of the field. This simple model ignores surface dark spots, which should be accounted for, see the text for details.

Brightness maps are produced as part of the magnetic imaging process, and for many accreting $\mathrm{T}$ Tauri stars the main magnetic pole often coincides with large dark spots (e.g. for BP Tau; Donati et al 2008b). Dark spots coinciding with the main magnetic pole will significantly change the respective contributions of the dipole and octupole field components to the longitudinal field curve, with the octupole producing a comparably larger contribution. If the cool spot is off-set from the main magnetic pole then the tilt of the dipole component $\beta_{d i p}$ that can be derived by fitting equation (15) to an observed longitudinal field curve will likely be significantly off.

If the longitudinal field curve constructed from a LSD average photospheric line was probing the large scale dipole component of a stellar magnetic field, then the variations of $B_{l o n}$ with phase would be mostly sinusoidal; observationally this is not usually the case. For V2129 Oph the departure from a simple sinusoidal longitudinal field curve is obvious, see the plot in figure 2 (bottom left panel) in Donati et al (2011a). For AA Tau the longitudinal field component measured from the LSD average photospheric absorption line is almost featureless, and yet detailed modeling of the full Stokes V profile reveals a strong dipole component of polar strength $\sim 2 \mathrm{kG}$. Longitudinal field components therefore provide poor observational constrains on $\mathrm{T}$ Tauri 
magnetic field topologies even for the dipole component itself.

Although strong and rotationally modulated longitudinal field components are often measured in accretion related emission lines (e.g. Valenti \& Johns-Krull 2004; Donati et al $2008 \mathrm{~b}$ ), there is another reason why fitting an oblique dipole rotator model to the longitudinal field curve may yield polar field strengths for the dipole component that are significantly discrepant from the true value. If we use V2129 Oph in July 2009 as a specific example, the dipole component recovered from a spherical harmonic decomposition of the magnetic map suggests a dipole component of $\sim 1 \mathrm{kG}$, but the bulk of the material arrives at the star into a single positive field spot of $\sim 4 \mathrm{kG}$ (Donati et al 2011a). Thus the analysis of the longitudinal field component measured in accretion related emission lines which are forming close to the accretion shock would yield discrepant values for the dipole component (on V2129 Oph higher order field components contribute to the strong field region at the base of the accretion funnel). Indeed Valenti \& Johns-Krull (2004) find that their longitudinal field components measured in the HeI $5876 \AA$ emission line are rotationally modulated, but well fitted by a simple model where the accreting material lands in a single polarity spot on the stellar surface. This is found in Zeeman-Doppler imaging studies for many accreting T Tauri stars (e.g. Donati et al 2008b; Donati et al 2011a). Analysis of the longitudinal field curve alone (whether derived from accretion related emission lines or from a LSD average photospheric absorption line) therefore provides poor constrains on the field topology and the polar strength of the dipole component for accreting $\mathrm{T}$ Tauri stars. A full analysis of variations in the Stokes V profile for both accretion related emission lines, and photospheric absorption lines, is clearly desirable whenever high resolution spectra are available to provide a consistent description of the large-scale magnetic field topology.

\section{Discussion and conclusions}

In this paper we have discussed the numerical field extrapolation and analytic models employed over the past few years to construct 3D models of the magnetospheres of accreting pre-main sequence stars. Each of the methods, analytic, field extrapolation, and also magnetohydrodynamic which we discuss below, have their own advantages, disadvantages and limitations. By writing out the field components for magnetospheres that consist of several high order field components analytically, as in section 3.2, results that may be missed by numerical models are revealed. As one example, if we consider the simplified case of a dipole plus octupole magnetic field, where the dipole and octupole moments are both aligned, then it becomes clear from equations (12) and (13) that a magnetic null point exists where $B_{r}=B_{\theta}=0$ at a radius of

$$
\frac{r_{n u l l}}{R_{*}}=\left(\frac{3}{4} \frac{B_{o c t}}{B_{d i p}}\right)^{1 / 2}
$$

$r_{n u l l} / R_{*}$ is the radius of circle in a plane tilted by $\beta$ (the tilts of the dipole and octupole moments) towards the phase that the multipole moments are tilted towards. Essentially this radius defines the point at where the dipole components begins to dominate the octupole component as we move away from the stellar surface towards larger radii. As the polar strength of the octupole relative to the polar strength of the dipole, $B_{o c t} / B_{d i p}$, is increased the extent of the region where the octupole component dominates increases and "smaller scale" field lines extending to greater height are found in the field extrapolations; this can be seen from the plots in Figure 6 for stars with larger $B_{o c t} / B_{d i p}$ ratios (see Table 1).

The major disadvantage of the analytic approach is the inclusion of a large amount of $\ell$ number multipoles rapidly becomes cumbersome and such models are typically (but not exclusively) limited to axisymmetric fields (e.g. Mohanty \& Shu 2008; Gregory et al 2010; Gregory 2011). Furthermore, when the multipole moments are tilted by different amounts relative to the stellar rotation axis, and/or towards different rotation phases, the field structure (i.e. the paths of the field lines exterior to the star) must be derived numerically from $B_{r} / \mathrm{d} r=B_{\theta} /(r \mathrm{~d} \theta)=B_{\phi} /(r \sin \theta \mathrm{d} \phi)$. Inclusion of non-axisymmetric field modes would further add to the complexity of the analytic work. In such cases models of the magnetospheres of stars with complex magnetic fields are best handled numerically.

Numerical field extrapolations from magnetic surface maps have the advantage over the analytic approach that as many $\ell$ and $m$ modes that can be resolved observationally can be included. Field extrapolation is currently the only method where magnetic fields with an observed degree of complexity can be handled and incorporated into models of the large-scale magnetosphere. Potential field models are computationally simple to carry out and only require desktop computing resources. They also produce unique field topologies (Aly 1987). However the field topologies obtained via potential field extrapolation are static. Time dependent effects, such as the distortion of the large scale magnetic field due to the interaction with the circumstellar disk, cannot be modeled. Other non-potential effects, such as flux emergence or surface differential rotation that has been measured for some accreting T Tauri stars (e.g. Donati et al 2010a, 2011a), also cannot be included.

Three-dimensional MHD models of the star-disk interaction with non-dipolar magnetic fields have also been developed by Long et al $(2007,2008,2009,2011)$ and Romanova et al (2011). The main advantage of the MHD approach is the ability to handle the time evolution of both the large-scale field, and the magnetospheric mass accretion process, that the potential field extrapolation models cannot. Unfortunately 3D MHD simulations require supercomputer resources; such models are therefore computationally, and fiscally, expensive. Likewise, magnetic maps have not yet been incorporated into MHD simulations as grid resolution issues arise. A small grid resolution close to the star is re- 
quired in order to include high order and non-axisymmetric field components, but at the same time, a large grid able to reach out as far as the inner disk is required. Instead the latest generation of 3D MHD models, presented by Romanova et al (2011) and Long et al (2011), begin by assuming that the star has a potential magnetic field consisting of a tilted dipole plus a tilted octupole component with field strengths and tilts being adopted from the published magnetic map of the star of interest. Such dipole-octupole field structures are then allowed to evolve due to the interaction with the disk. The largest scale field lines are torn open and quickly twisted around the rotation axis of the star, see e.g. figure 6 of Romanova et al (2011), however, the field interior to this that carries gas in columns on to the star retains its initial potential structure.

MHD models that incorporate the magnetic maps derived from Zeeman-Doppler imaging remain to be carried out in the future. Likewise a major new avenue for research is the inclusion of stellar surface differential rotation, flux emergence and meridional flows into MHD models. It may turn out that the retention of the potential field structure found in current simulations is no longer valid once stellar surface transport effects have been accounted for. Likewise it is unclear whether $\mathrm{T}$ Tauri stars undergo magnetic cycles, although there is tentative evidence that they do and that their large scale magnetospheres evolve over long (possibly several years) timescales (e.g. Donati et al 2011a).

The veracity of the 3D field structures obtained via field extrapolation from the magnetic maps must continue to be examined in future. On going large multi-wavelength programs are useful here (e.g. Argiroffi et al 2011; Donati et al 2011c). Based on a field extrapolation model of V2129 Oph Jardine et al (2008), using a simple accretion flow model presented in Gregory et al (2007), predicted that a clear signature of accretion related X-ray emission would be detected, which was subsequently found (Argiroffi et al 2011). Never-the-less, further tests are underway to determine if the derived field topologies genuinely capture the true magnetospheric structure of T Tauri stars.

Acknowledgements. SGG thanks the organizers for the invitation to give an invited review talk at the meeting, and for financial support. We thank Chris Johns-Krull \& Wei Chen for discussions regarding the stellar-disk averaged longitudinal magnetic field component, and Fred Adams \& Sean Matt for many interesting discussions. SGG is supported by NASA grant HST-GO-11616.07A. The "Magnetic Protostars and Planets" (MaPP) project is supported by the funding agencies of CFHT and TBL (through the allocation of telescope time) and by CNRS/INSU in particular, as well as by the French "Agence Nationale pour la Recherche" (ANR).

\section{A Tilted multipole moments in 3D}

We consider two Cartesian coordinate systems (i) the stellar reference frame $(x, y, z)$ where the stellar rotation axis is aligned with the $z$-axis $(\boldsymbol{\Omega}=\Omega \hat{\mathbf{z}})$ and (ii) the multipole reference frame $\left(x^{\prime}, y^{\prime}, z^{\prime}\right)$ where the multipole moment symmetry axis is aligned
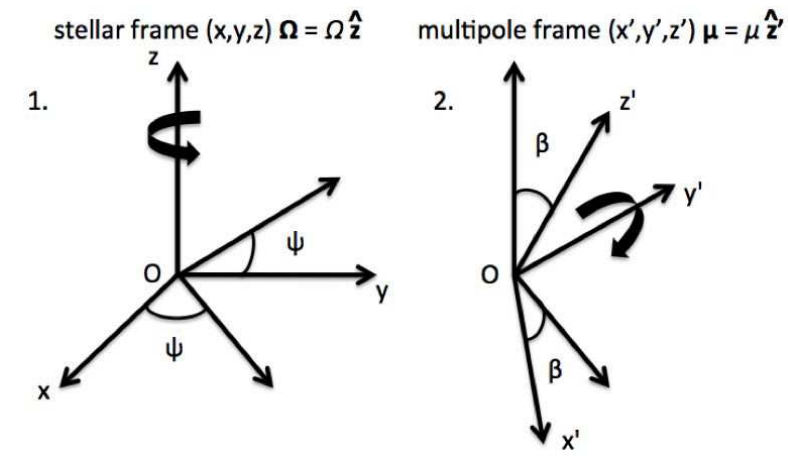

Fig. A1 A given multipole moment symmetry axis $\boldsymbol{\mu}$ is assumed to be tilted by an angle $\beta$, relative to the stellar rotation axis, towards a longitude $\psi$, where $\psi$ is related to the rotation phase via $\psi=(1-$ phase $) \times 360^{\circ}$, assuming the $x z$-plane corresponds to a rotation phase of zero. The stellar reference frame $(x, y, z)$ is defined such that $\boldsymbol{\Omega}=$ $\Omega \hat{\mathbf{z}}$, and the multipole frame $\left(x^{\prime}, y^{\prime}, z^{\prime}\right)$ such that $\boldsymbol{\mu}=\mu \hat{\mathbf{z}^{\prime}}$. We can transform from the stellar coordinate frame to the multipole frame by 1 . rotating the stellar coordinate system by $\psi$ around the $z$-axis, followed by 2 . a rotation about the new $y$-axis by an angle $\beta$ as illustrated.

with the $z^{\prime}$-axis $\left(\boldsymbol{\mu}=\mu \hat{\mathbf{z}^{\prime}}\right)$. For an arbitrary $\ell$-number multipole Gregory et al. (2010) showed that the magnitude of the multipole moment is related to the polar strength of the multipole via,

$$
\mu=R_{*}^{\ell+2} B_{*}^{\ell, \text { pole }} /(\ell+1) .
$$

We assume that the $x z$-plane corresponds to a rotation phase of zero. The multipole moment is assumed to be tilted by an angle $\beta$, such that $\hat{\boldsymbol{\Omega}} \cdot \hat{\boldsymbol{\mu}}=\cos \beta$, towards a phase determined by another angle $\psi$, the longitude in the stellar reference frame, and where $\hat{\boldsymbol{\Omega}}$ and $\hat{\boldsymbol{\mu}}$ are unit vectors along $\boldsymbol{\Omega}$ and $\boldsymbol{\mu}$ respectively. In other words $\beta=45^{\circ}$ and $\psi=90^{\circ}$, represents a multipole moment tilted by $45^{\circ}$ from the stellar rotation axis, towards rotation phase 0.75 i.e. $\psi=(1-$ phase $) \times 360^{\circ}$. Thus in order to transform from the coordinate system that denotes the frame of the star to that which denotes the frame of the multipole we first rotate counterclockwise about the $z$-axis (when looking towards the origin) by an angle $\psi$, and then counterclockwise by an angle $\beta$ (when looking towards the origin) about the new $y$-axis, as illustrated in Figure A1 (note that we are rotating the entire coordinate system, and not simply a vector within the same fixed coordinate system). The Cartesian components of the multipole moment in the stellar reference frame, where $\boldsymbol{\mu}=\mu_{x} \hat{\mathbf{x}}+\mu_{y} \hat{\mathbf{y}}+\mu_{z} \hat{\mathbf{z}}$, are

$$
\begin{aligned}
& \left(\begin{array}{l}
\mu_{x} \\
\mu_{y} \\
\mu_{z}
\end{array}\right)= \\
& \left(\begin{array}{ccc}
\cos \psi & -\sin \psi & 0 \\
\sin \psi & \cos \psi & 0 \\
0 & 0 & 1
\end{array}\right)\left(\begin{array}{ccc}
\cos \beta & 0 & \sin \beta \\
0 & 1 & 0 \\
-\sin \beta & 0 & \cos \beta
\end{array}\right)\left(\begin{array}{l}
0 \\
0 \\
\mu
\end{array}\right) .
\end{aligned}
$$

The vector components of $\boldsymbol{\mu}$ can then be converted from the Cartesian to a spherical coordinate system $(r, \theta, \phi)$ where $r=0$ corresponds to the center of the star, $\phi=0$ corresponds to the stellar $x z$-plane and $\theta$ is the co-latitude in the stellar reference frame. 
Noting that $\boldsymbol{\mu}=\mu_{x} \hat{\mathbf{x}}+\mu_{y} \hat{\mathbf{y}}+\mu_{z} \hat{\mathbf{z}}=\mu_{r} \hat{\mathbf{r}}+\mu_{\theta} \hat{\boldsymbol{\theta}}+\mu_{\phi} \hat{\boldsymbol{\phi}}$, then

$$
\left(\begin{array}{l}
\mu_{r} \\
\mu_{\theta} \\
\mu_{\phi}
\end{array}\right)=\left(\begin{array}{ccc}
\sin \theta \cos \phi & \sin \theta \sin \phi & \cos \theta \\
\cos \theta \cos \phi & \cos \theta \sin \phi & -\sin \theta \\
-\sin \phi & \cos \phi & 0
\end{array}\right)\left(\begin{array}{l}
\mu_{x} \\
\mu_{y} \\
\mu_{z}
\end{array}\right)
$$

The spherical components of the tilted multipole moment in the stellar reference frame are thus given by equations 6 .

\section{B Stellar disk averaged longitudinal magnetic field component}

Goossens (1979) derived an expression for the stellar disk averaged longitudinal magnetic field component $B_{l o n}$ arising from a multipolar stellar magnetic field, see his equation (20) with the ' $W$ ' terms for a linear limb darkening law from his equations (25a-d) see also Stibbs (1950) and Schwarzschild (1950). It is applicable to the case where all of the multipole moments are aligned with each other, and which are viewed at an angle $\alpha$ to the magnetic axis. The first term in Goossens' equation (20) is the dipole term, the second term is the quadrupole term, the third term represents the contribution from the higher order even $\ell$ number multipoles ( $\ell=4$ [hexadecapole], $6,8 \ldots$ ), and the fourth term is the contribution from the higher order odd $\ell$ number multipoles $(\ell=3$ [octupole], 5 [dotriacontapole], 7 ...). Goossens then goes on to derive the stellar disk averaged longitudinal field component for a dipole-quadrupole magnetic field, but this can be extended to any arbitrary combination of high order $\ell$ number multipoles.

By comparing the magnetostatic potential in Goossens (1979) [his equation (4)] with that used in Gregory et al. (2010) [their equations (3.18) and (3.27)], the 'A' terms in Goossens (1979) equation (20) are related to the polar field strengths of the particular multipoles via,

$$
A_{n}=M_{\ell}=\frac{1}{(\ell+1)} R_{*}^{\ell+2} B_{*}^{\ell, \text { pole }},
$$

where $B_{*}^{\ell, \text { pole }}$ is the polar field strength of the $\ell$ th multipole, and we rewrite $B_{*}^{1, \text { pole }} \equiv B_{\text {dip }}, B_{*}^{2, \text { pole }} \equiv B_{\text {quad }}$, and $B_{*}^{3, \text { pole }} \equiv$ $B_{\text {oct }}$ for brevity. Thus, the 'A' terms in Goossens equation (20) can be re-written as

$$
\begin{aligned}
A_{1} & =\frac{1}{2} R_{*}^{3} B_{\text {dip }} \\
A_{2} & =\frac{1}{3} R_{*}^{4} B_{\text {quad }} \\
A_{2 k} & =\frac{1}{(2 k+1)} R_{*}^{2 k+2} B_{*}^{2 k, \text { pole }} \\
A_{2 k+1} & =\frac{1}{(2 k+2)} R_{*}^{2 k+3} B_{*}^{2 k+1, \text { pole }}
\end{aligned}
$$

where the first term is the dipole term, the second the quadrupole term, the third term the higher order even $\ell$ number multipoles, and the fourth term the higher order odd $\ell$ number multipoles. Equation (20) of Goossens can then be re-expressed by replacing the 'A' and 'W' terms,

$$
\begin{aligned}
B_{\text {lon }}= & \frac{1}{20} B_{\text {dip }} \frac{(15+u)}{(3-u)} P_{1}(\cos \alpha) \\
& +\frac{1}{4} B_{\text {quad }} \frac{u}{(3-u)} P_{2}(\cos \alpha) \\
& +\frac{3 u}{(3-u)} \sum_{k=2}^{\infty} C_{k} \frac{B_{*}^{2 k, \text { pole }}}{(2 k+1)} P_{2 k}(\cos \alpha) \\
& +\frac{3(1-u)}{(3-u)} \sum_{k=1}^{\infty} D_{k} \frac{B_{*}^{2 k+1, \text { pole }}}{(2 k+2)} P_{2 k+1}(\cos \alpha)
\end{aligned}
$$

where $P_{j}(\cos \alpha)$ are the Legendre polynomials, $\alpha$ is the angle between the magnetic axis (here it is assumed all the multipole moments are aligned with each other) and the observer's line-of-sight, and

$$
\begin{aligned}
& \begin{aligned}
C_{k} & =(-1)^{k+1} \frac{(2 k+1)(2 k-3) ! !}{2^{k}(k+2) !} \\
D_{k} & =(-1)^{k} \frac{(k+1)(2 k-1) ! !}{2^{k}(k+2) !} \\
\text { with }(2 k-1) ! ! & =(2 k-1)(2 k-3)(2 k-5) \ldots 5 \times 3 \times 1 .
\end{aligned}
\end{aligned}
$$

\section{B.1 Dipole-octupole longitudinal magnetic field component}

The dipole longitudinal field component is the first term of equation (B1), and derivations can be found in Schwarzschild (1950) and Stibbs (1950). The octupole magnetic field term is the $k=1$ term in the final summation term of equation B1]. Thus the disk averaged longitudinal magnetic field component for a pure octupole is,

$$
\begin{aligned}
B_{\text {lon }}^{\text {oct }} & =\frac{1}{8} B_{\text {oct }} \frac{(u-1)}{(3-u)} P_{3}(\cos \alpha) \\
& =\frac{1}{16} B_{\text {oct }} \frac{(u-1)}{(3-u)}\left(5 \cos ^{3} \alpha-3 \cos \alpha\right)
\end{aligned}
$$

where $\alpha$ is the angle between the octupole moment and the observer's line-of-sight. $\alpha$ is related to the stellar inclination $i$ and the angle between the stellar rotation pole and the multipole moment $\beta$ via,

$$
\cos \alpha=\cos i \cos \beta+\sin i \sin \beta \cos \phi
$$

where $\phi$ is the azimuthal angle (the longitude) of the magnetic axis at some time $t$ i.e. $\phi=2 \pi(1-\Phi)$ where $\Phi$ is the rotation phase (see the discussion in Goossens (1979) around his equations (23) and (24)). Equation (B2) can be derived by considering the circle swept out by the magnetic axis rotating about the stellar rotation axis (it is just the spherical cosine law). If at time $t=0$, i.e. rotation phase $\Phi=0$, the magnetic axis is tilted towards some rotation phase $\Phi_{0}$ then $\cos \phi=\cos \left(2 \pi[1-\Phi]+2 \pi\left[1-\Phi_{0}\right]\right)=$ $\cos \left(2 \pi\left[\Phi-\Phi_{0}\right]\right)$, thus,

$$
\cos \alpha=\cos i \cos \beta+\sin i \sin \beta \cos \left(2 \pi\left[\Phi-\Phi_{0}\right]\right) .
$$

The stellar disk averaged longitudinal magnetic field component for an octupole is then,

$$
\begin{aligned}
& B_{\text {lon }}^{\text {oct }}(\Phi)=\frac{1}{16} B_{\text {oct }}\left(\frac{u-1}{3-u}\right) \times \\
& \left\{5\left(\cos \beta_{\text {oct }} \cos i+\sin \beta_{o c t} \sin i \cos \left[2 \pi\left(\Phi-\Phi_{o c t}\right)\right]\right)^{3}\right. \\
& \left.-3\left(\cos \beta_{o c t} \cos i+\sin \beta_{o c t} \sin i \cos \left[2 \pi\left(\Phi-\Phi_{o c t}\right)\right]\right)\right\} .
\end{aligned}
$$

Goossens (1979) consider the dipole-quadrupole case where the dipole and quadrupole moments are tilted by different amounts towards different rotation phases (there is a $\sin i$ term missing in the second term of his equation (35)). The Goossens work is straightforward to extend to dipole-octupole magnetic fields, with the dipole and octupule tilted towards different rotation phases and by different amounts relative to the stellar rotation axis. Taking the dipole and octupole components of equation B1

$$
\begin{aligned}
B_{\text {lon }}(\Phi) & =\frac{1}{20} B_{\text {dip }} \frac{(15+u)}{(3-u)} \cos \alpha_{\text {dip }} \\
& +\frac{1}{16} B_{\text {oct }} \frac{(u-1)}{(3-u)}\left(5 \cos ^{3} \alpha_{\text {oct }}-3 \cos \alpha_{\text {oct }}\right)(\mathrm{B} 5)
\end{aligned}
$$


where $\alpha_{\text {dip }}$ and $\alpha_{\text {oct }}$ are the angles between the dipole moment and the stellar rotation axis, and the octupole moment and the stellar rotation axis, respectively. In the notation adopted by Goossens, see their equation (35), $\lambda$ is the angle between the plane that contains the dipole moment and the stellar rotation axis, and the plane that contains the quadrupole moment (octupole moment in our case) and the stellar rotation axis. Thus, in the Goossens notation, at time $t=0$ if the dipole moment is titled towards longitude $\phi_{0}$ then the octupole (or quadrupole in the Goossens paper) moment is tilted towards longitude $\phi_{0}+\lambda$; in other words we can write,

$$
\begin{aligned}
& \cos \alpha_{d i p}= \\
& \cos i \cos \beta_{d i p}+\sin i \sin \beta_{d i p} \cos \left(2 \pi\left[\Phi-\Phi_{d i p}\right]\right) \\
& \cos \alpha_{o c t}= \\
& \cos i \cos \beta_{\text {oct }}+\sin i \sin \beta_{\text {oct }} \cos \left(2 \pi\left[\Phi-\Phi_{\text {oct }}\right]\right) .
\end{aligned}
$$

Putting the equations in this section together gives equation (15), the general result for the dipole-octupole stellar disk averaged longitudinal magnetic field component as a function of rotation phase.

\section{References}

Adams, F. C., Gregory, S. G.: 2011, ApJ, in press [astro$\mathrm{ph} / 1109.5948]$

Altschuler, M. D., Newkirk, G.: 1969, Solar Physics 9, 131

Aly, J. J.: 1987, Solar Physics 111, 287

Argiroffi, C., Flaccomio, E., Bouvier, J., et al.: 2011, A\&A 530, A1

Aurière, M.: 2003, EAS Publications Series 9, 105

Bagnulo, S., Landi Degl'Innocenti, M., Landi Degl'Innocenti, E.: 1996, A\&A 308, 115

Bessolaz, N., Zanni, C., Ferreira, J., Keppens, R., Bouvier, J.: 2008, A\&A 478, 155

Collier Cameron, A., Campbell, C. G.: 1993, A\&A 274, 309

Donati, J.-F., Semel, M., Carter, B. D., Rees, D. E., Collier Cameron, A.: 1997, MNRAS 291, 658

Donati, J.-F.: 2001, Astrotomography, Indirect Imaging Methods in Observational Astronomy, Lecture Notes in Physics 573, 207

Donati, J.-F., Collier Cameron, A., Semel, M., et al.: 2003, MNRAS 345, 1145

Donati, J.-F., Catala, C., Landstreet, J. D., Petit, P.: 2006a, Astronomical Society of the Pacific Conference Series 358, 362

Donati, J.-F., Howarth, I. D., Jardine, M. M., et al.: 2006b, MNRAS 370, 629

Donati, J.-F., Jardine, M. M., Gregory, S. G., et al.: 2007, MNRAS 380, 1297

Donati, J.-F., Morin, J., Petit, P., et al.: 2008a, MNARS 390, 545

Donati, J.-F., Jardine, M. M., Gregory, S. G., et al.: 2008b, MNRAS 386, 1234

Donati, J.-F., Landstreet, J. D.: 2009, ArA\&A 47, 333

Donati, J.-F., Skelly, M. B., Bouvier, J., et al.: 2010a, MNRAS 402, 1426

Donati, J.-F., Skelly, M. B., Bouvier, J., et al.: 2010b, MNRAS 409, 1347

Donati, J.-F., Bouvier, J., Walter, F. M., et al.: 2011a, MNRAS 412, 2454

Donati, J.-F., Gregory, S. G., Alencar, S. H. P., et al.: 2011b, MNRAS 417, 472

Donati, J.-F., Gregory, S. G., Montmerle, T., et al.: 2011c, MNRAS 417,1747

Dunstone, N. J., Hussain, G. A. J., Collier Cameron, A., et al.: 2008, MNRAS 387, 1525
Flaccomio, E., Micela, G., Sciortino, S., et al.: 2005, ApJS 160, 450

Goodson, A. P., Winglee, R. M., Böhm, K.-H.: 1997 ApJ, 489, 199

Goossens, M.: 1979, A\&A 79, 210

Gregory, S. G., Jardine, M., Collier Cameron, A., Donati, J.-F.: 2005, 13th Cambridge Workshop on Cool Stars, Stellar Systems and the Sun, ESA SP 560, 191

Gregory, S. G., Jardine, M., Simpson, I., Donati, J.-F.: 2006a, MNRAS 371, 999

Gregory, S. G., Jardine, M., Collier Cameron, A., Donati, J.-F.: 2006b, MNRAS 373, 827

Gregory, S. G., Wood, K., Jardine, M.: 2007, MNRAS 379, L35

Gregory, S. G., Matt, S. P., Donati, J.-F., Jardine, M.: 2008, MNRAS 389, 1839

Gregory, S. G., Jardine, M., Gray, C. G., Donati, J.-F.: 2010, Reports on Progress in Physics 73, 126901

Gregory, S. G.: 2011, American Journal of Physics 79, 461

Gregory, S. G., Donati, J.-F., Morin, J., Hussain, G. A. J., Mayne, N., Hillenbrand, L. A., Jardine, M.: 2012, ApJ submitted

Hartmann, L., Hewett, R., Calvet, N.: 1994, ApJ 426, 669

Hussain, G. A. J., Jardine, M., Donati, J.-F., et al.: 2007, MNRAS 377,1488

Hussain, G. A. J., Collier Cameron, A., Jardine, M. M., et al.: 2009, MNRAS 398, 189

Jardine, M., Collier Cameron, A., Donati, J.-F.: 2002, MNRAS 333, 339

Jardine, M., Collier Cameron, A., Donati, J.-F., Gregory, S. G., Wood, K.: 2006, MNRAS 367, 917

Jardine, M. M., Gregory, S. G., Donati, J.-F.: 2008, MNRAS 386, 688

Johns-Krull, C. M., Valenti, J. A., Hatzes, A. P., Kanaan, A.: 1999, ApJL 510, L41

Johns-Krull, C. M.: 2007, ApJ 664, 975

Johnstone, C., Jardine, M., Mackay, D. H.: 2010, MNRAS 404 101

Königl, A.: 1991, ApJL 370, L39

Küker, M., Henning, T., Rüdiger, G.: 2003, ApJ 589, 397

Kurosawa, R., Harries, T. J., Symington, N. H.: 2006, MNRAS 370,580

Loinard, L., Torres, R. M., Mioduszewski, A. J., Rodríguez, L. F.: 2008, ApJL 675, L29

Long, M., Romanova, M. M., Lovelace, R. V. E.: 2007, MNRAS 374,436

Long, M., Romanova, M. M., Lovelace, R. V. E.: 2008, MNRAS 386,1274

Long, M., Romanova, M. M., Lamb, F. K.: 2009, submitted [astro$\mathrm{ph} / 0911.5455]$

Long, M., Romanova, M. M., Kulkarni, A. K., Donati, J.-F.: 2011, MNRAS 413, 1061

Marsden, S. C., Jardine, M. M., Ramírez Vélez, J. C., et al.: 2011, MNRAS 413, 1939

Matt, S., Pudritz, R. E.: 2005, MNRAS 356, 167

Miller, K. A., Stone, J. M.: 1997, ApJ 489, 890

Mohanty, S., Shu, F. H.: 2008, ApJ 687, 1323

Morin, J., Donati, J.-F., Petit, P., et al.: 2008, MNRAS 390, 567

Morin, J., Donati, J.-F., Petit, P., et al.: 2010, MNRAS 407, 2269

Morin, J., Dormy, E., Schrinner, M., Donati, J.-F.: 2011, MNRAS submitted [astro-ph/1106.4263]

Orlando, S., Reale, F., Peres, G., Mignone, A.: 2011, MNRAS 415, 3380

Petrov, P. P., Gahm, G. F., Stempels, H. C., Walter, F. M., Artemenko, S. A.: 2011, A\&A in press [astro-ph/1109.1266]

Preston, G. W.: 1967, ApJ 150, 547 
Riley, P., Linker, J. A., Mikić, Z., et al.: 2006, ApJ 653, 1510

Romanova, M. M., Ustyugova, G. V., Koldoba, A. V., Lovelace, R. V. E.: 2002, ApJ 578, 420

Romanova, M. M., Ustyugova, G. V., Koldoba, A. V., Wick, J. V., Lovelace, R. V. E.: 2003, ApJ 595, 1009

Romanova, M. M., Ustyugova, G. V., Koldoba, A. V., Lovelace, R. V. E.: 2004, ApJ 610, 920

Romanova, M. M., Long, M., Lamb, F. K., Kulkarni, A. K., Donati, J.-F.: 2011, MNRAS 411, 915

Ruan, P., Wiegelmann, T., Inhester, B., et al.: 2008, A\&A 481, 827

Safier, P. N.: 1998, ApJ 494, 336

Schatten, K. H., Wilcox, J. M., Ness, N. F.: 1969, Solar Physics 6, 442

Schulz, M., Frazier, E. N., Boucher, D. J., Jr.: 1978, Solar Physics 60,83

Schwarzschild, M.: 1950, ApJ 112, 222

Shu, F., Najita, J., Ostriker, E., et al.: 1994, ApJ 429, 781

Skelly, M. B., Donati, J.-F., Bouvier, J., et al.: 2011, MNRAS submitted

Stibbs, D. W. N.: 1950, MNRAS 110, 395

Valenti, J. A., Johns-Krull, C. M.: 2004, Ap\&SS 292, 619

von Rekowski, B., Piskunov, N.: 2006, Astronomische Nachrichten 327, 340

Yang, H., Johns-Krull, C. M.: 2011, ApJ 729, 83

Zanni, C., Ferreira, J.: 2009, A\&A 508, 1117 